Publ. RIMS, Kyoto Univ.

15 (1979), 357-399

\title{
A Characterization of the Hyperbolic Mixed Problems in a Quarter Space for Differential Operators with Constant Coefficients
}

By

\author{
Yoshihiro SHIBATA*
}

\section{$\S 1$. Introduction}

L. Gårding showed in his papers [2] and [3] that the following three statements on a differential operator $P(D)$ with constant coefficients are equivalent (see also Atiyah, Bott and Gårding [1]).

(I) The Cauchy problem for $P(D)$ in the half space $\left\{x \in \boldsymbol{R}^{n} ; x \cdot \vartheta>0\right\}$ with data on the plane $x \cdot \vartheta=0$ is $C^{\infty}$ (or $\mathcal{E}$ ) well posed, where $\vartheta$ is a non-zero vector in the real dual $\boldsymbol{\xi}^{n}$ of the real $n$-dimensional Euclidean space $\boldsymbol{R}^{n}$ by the inner product $x \cdot \xi=x_{1} \cdot \xi_{1}+\cdots+x_{n} \cdot \xi_{n}$.

(II) $P(D)$ has a fundamental solution $E$ satisfying $P(D) E=\delta$ and having support in some closed proper $^{1)}$ cone $K$ with its vertex at the origin such that $x \cdot \vartheta>0$ on $K-\{0\}$.

(III) The characteristic polynomial $P(\xi)$ of the operator $P(D)$ has the properties:

(1. 1) $P^{0}(\vartheta) \neq 0$ where $P^{0}(\xi)$ is the principal part of $P(\xi)$,

and

(1.2) $P(\xi+s \vartheta) \neq 0$ when $\xi \in \mathbb{E}^{n}$ and $\operatorname{Im} s$ is less than some fixed number $\gamma_{0}$.

Such a differential operator $P(D)$ (resp. a polynomial $P(\xi)$ ) is said to be hyperbolic with respect to $\vartheta$.

Now consider the mixed initial-boundary value problem in the quarter space $\left\{x=\left(x_{1}, \cdots, x_{n}\right) \in \boldsymbol{R}^{n} ; x_{1}>0, x_{n}>0\right\}$ for a system $\left\{P(D) ; B_{j}(D)\right.$,

Communicated by S. Matsuura, March 28, 1977.

* Institute of Mathematics, The University of Tsukuba.

1) A cone which does not contain any straight lines is said to be proper. 
$j=1, \cdots, q\}$ of differential operators with constant coefficients:

$$
\begin{aligned}
& P(D) u(x)=f(x), \quad x_{1}>0, x_{n}>0, \\
& \left.D_{1}^{j} u(x)\right|_{x_{1}=0}=g_{j}\left(x^{\prime \prime}\right), \quad j=0,1, \cdots, m-1, x_{n}>0, \\
& \left.B_{j}(D) u(x)\right|_{x_{n}=0}=h_{j}\left(x^{\prime}\right), \quad j=1, \cdots, q, x_{1}>0,
\end{aligned}
$$

where $m$ is the order of $P(D)$. Then the corresponding question for the system $\left\{P(D) ; B_{j}(D), j=1, \cdots, q\right\}$ can be proposed. After the works [5], [6] by R. Hersh and [11] by T. Shirota, R. Sakamoto [9] proved the following.

Assume that $P(\xi)$ is a hyperbolic polynomial with respect to $\vartheta=(1,0, \cdots, 0)$ and the boundary $\partial \boldsymbol{R}_{+}^{n}=\left\{x \in \boldsymbol{R}_{+}^{n} ; x_{n}=0\right\} \quad\left(\boldsymbol{R}_{+}^{n}=\left\{x=\left(x_{1}\right.\right.\right.$, $\left.\left.\left.\cdots, x_{n}\right) \in \boldsymbol{R}^{n} ; x_{n}>0\right\}\right)$ is non-characteristic for $P(D)$ and $B_{j}(D) \quad(j=1$, $\cdots, q)$ and that $q$ is equal to the constant number $p$ of the roots with positive imaginary part of the equation $P\left(\xi^{\prime}-i s \vartheta^{\prime}, \lambda\right)=0$ in $\lambda$ with $s$ large enough where $\xi^{\prime}=\left(\xi_{1}, \cdots, \xi_{n-1}\right)$. Then the mixed problem (1.3)(1.5) is $C^{\infty}$ (or $\mathcal{E}$ ) well posed if and only if the Lopatinski determinant $R\left(\zeta^{\prime}\right)^{2)}$ of $\left\{P(D) ; B_{j}(D), j=1, \cdots, p\right\}$ has the properties corresponding to $(1.1)$ and (1.2):

(1.6) $R^{0}\left(\vartheta^{\prime}\right) \neq 0$, where $R^{0}\left(\zeta^{\prime}\right)$ is the principal part of $R\left(\zeta^{\prime}\right)$.

(1.7) $R\left(\xi^{\prime}+s \vartheta^{\prime}\right) \neq 0$ when $\xi^{\prime} \in \boldsymbol{\Xi}^{n-1}$ and $\operatorname{Im} s$ is less than some fixed number.

Such a Lopatinski determinant $R\left(\zeta^{\prime}\right)$ is said to be hyperbolic with respect to $\vartheta^{\prime}$. Moreover she showed the existence of a unique system $\{E(x, y)$; $\left.F_{j}(x), j=1, \cdots, p\right\}$ of fundamental solutions having support in some closed proper cones.

A system $\left\{E(x, y) ; F_{j}(x), j=1, \cdots, q\right\}$ of fundamental solutions of the mixed problems (1.3)-(1.5) for $\left\{P(D) ; B_{j}(D), j=1, \cdots, q\right\}$ is defined as a system of solutions of the following equations such that $E(x, y) \in \mathscr{D}^{\prime}\left(\boldsymbol{R}_{+}^{n}\right) \cap C^{\infty}\left(\left[0, y_{n}\right) ; \mathscr{D}^{\prime}\left(\boldsymbol{R}^{n-1}\right)\right)^{3)} \cap C^{\infty}\left(\left(y_{n}, \infty\right) ; \mathscr{D}^{\prime}\left(\boldsymbol{R}^{n-1}\right)\right)$ and $F_{j}(x), j=1, \cdots, q$, belong to $C^{\infty}\left([0, \infty) ; \mathscr{D}^{\prime}\left(\boldsymbol{R}^{n-1}\right)\right) \subset \mathscr{D}^{\prime}\left(\overline{\boldsymbol{R}_{+}^{n}}\right)$.

$$
P\left(D_{x}\right) E(x, y)=\delta(x-y), x_{n}>0, y=\left(0, y^{\prime \prime}\right) \in \boldsymbol{R}_{+}^{n},
$$

2) This will be defined in Section 4.

3) This space will be defined in Section 3. 


$$
\begin{aligned}
& \left.B_{j}\left(D_{x}\right) E(x, y)\right|_{x_{n}=0}=0, \quad j=1, \cdots, q, \\
& P(D) F_{k}(x)=0, \quad x_{n}>0, \quad k=1, \cdots, q, \\
& \left.B_{j}(D) F_{k}(x)\right|_{x_{n}=0}=\delta_{j k}\left(x^{\prime}\right), \quad j, k,=1, \cdots, q,
\end{aligned}
$$

where $\delta$ is the Dirac delta function, $y^{\prime \prime}=\left(y_{2}, \cdots, y_{n}\right), x^{\prime}=\left(x_{1}, \cdots, x_{n-1}\right)$ and $\delta_{j k}$ is the Kronecker delta symbol (i.e., $\delta_{j k}=0$ if $j \neq k$ and $\delta_{j k}=1$ if $j=k) . \quad D_{j}$ denotes $i^{-1} \partial / \partial x_{j}$ and $D$ denotes $\left(D_{1}, \cdots, D_{n}\right)$.

We say that a system $\left\{P(D) ; B_{j}(D), j=1, \cdots, q\right\}$ of differential operators is hyperbolic in $\boldsymbol{R}_{+}^{n}$ with respect to $\vartheta=(1,0, \cdots, 0)$ if it has a unique system $\left\{E(x, y) ; F_{j}(x), j=1, \cdots, q\right\}$ of fundamental solutions of the mixed problem (1.3)-(1.5) such that the support of $E(x, y)$ with respect to $x$ is contained in $(K+y) \cap \overline{\boldsymbol{R}_{+}^{n}}$ and the supports of the $F_{k}(x)$ are contained in $K \cap \overline{\boldsymbol{R}_{+}^{n}}$. Here $K$ is a closed proper cone with its vertex at the origin such that $x \cdot \vartheta>0$ on $K-\{0\}$.

The purpose of this paper is to characterize the hyperbolicity for the mixed problem (1.3)-(1.5) by showing that $\left\{P(D) ; B_{j}(D), j=1\right.$, $\cdots, q\}$ is hyperbolic in $\boldsymbol{R}_{+}^{n}$ with respect to $\vartheta=(1,0, \cdots, 0)$ if and only if $P(\xi)$ and $R\left(\zeta^{\prime}\right)$ are both hyperbolic with respect to $\vartheta$ and $\vartheta^{\prime}$, respectively. Note that we do not assume that the hyperplane $x_{n}=0$ is non-characteristic for $P(D)$ and $B_{j}(D)$. We shall show

Main Theorem. If a system $\left\{P(D) ; B_{j}(D), j=1, \cdots, q\right\}$ of differential operators is hyperbolic in $\boldsymbol{R}_{+}^{n}$ with respect to $\vartheta=(1,0, \cdots, 0)$, then the characteristic polynomial $P(\xi)$ has the properties (1.1) and (1.2) with respect to $\vartheta$ and the number $q$ of the boundary operators $B_{j}(D)$ must be equal to the constant number $p$ of the roots with positive imaginary part of the equation $P\left(\xi^{\prime}-i s \vartheta^{\prime}, \lambda\right)=0$ in $\lambda$ with $s$ large enough and the Lopatinski determinant $R\left(\zeta^{\prime}\right)$ has the properties (1.6) and (1.7).

The plan of the remainder is as follows. In Section 2 we prove the hyperbolicity of the characteristic polynomial $P(\xi)$ with respect to $\vartheta$. We give some preliminaries for the latter sections in Section 3. In Section 4 we prove the hyperbolicity of the Lopatinski determinant with respect to $\vartheta^{\prime}$ under the assumption $p=q \geq 1$. In Section 5 we complete our proof 
of the main theorem. In Section 6 the system of fundamental solutions $\left\{E(x, y) ; F_{j}(x), j=1, \cdots, q\right\}$ are constructed under the assumptions:

(I) $P(\xi)$ is hyperbolic with respect to $\vartheta=(1,0, \cdots, 0)$,

(II) $p=q$,

(III) The Lopatinski determinant of the system $\left\{P(D) ; B_{j}(D)\right.$, $j=1, \cdots, p\}$ is hyperbolic with respect to $\vartheta^{\prime}$.

Moreover we shall prove that $E(x, y)$ belongs to $C^{\infty}\left(\left[0, y_{n}\right)\right.$; $\left.\mathscr{D}^{\prime}\left(\boldsymbol{R}^{n-1}\right)\right) \cap C^{\infty}\left(\left(y_{n}, \infty\right) ; \mathscr{D}^{\prime}\left(\boldsymbol{R}^{n-1}\right)\right)$ and $F_{j}(x)$ belongs to $C^{\infty}([0, \infty)$; $\left.\mathscr{D}^{\prime}\left(\boldsymbol{R}^{n-1}\right)\right), j=1, \cdots, q$ and that such a system is unique. When the boundary $x_{n}=0$ is non-characteristic for $P(D)$, we give a proof of the uniqueness of this system. The proof is done by using the Paley-WienerSchwartz theorem. When the condition is not assumed, we prove it by a standard method employing the adjoint system of $\left\{P(D) ; B_{j}(D)\right.$, $j=1, \cdots, p\}$ and by modifying the proof given in [9].

The author would like to express his sincere gratitude to Professor M. Matsumura for many valuable suggestions and much kind encouragement and also to Professor S. Wakabayashi for his valuable advice. The proof of Proposition 3.1 in Section 3 is due to Professor S. Wakabayashi.

\section{$\S 2$. Hyperbolicity of the Characteristic Polynomial}

In this section we shall prove that the existence of certain fundamental solution $E(x, y)$ implies hyperbolicity of the characteristic polynomial $P(\xi)$. This can be done by a slight modification of the proof given in Atiyah, Bott and Gårding [1].

Proposition 2. 1. Assume that for some $y=\left(0, y^{\prime \prime}\right) \in \boldsymbol{R}_{+}^{n}$ there exists a fundamental solution $E(x, y)$ of the mixed problem (1.3)(1.5) (i.e., solution of the equations (1.8) and (1.9)) such that the support of $E(x, y)$ with respect to $x$ is contained in $(K+y) \cap \overline{\boldsymbol{R}_{+}^{n}}$. Here $K$ is a closed proper cone with its vertex at the origin such that $x \cdot \vartheta>0$ on $K-\{0\}$. Then the characteristic polynomial $P(\xi)$ is hyperbolic with respect to $\vartheta=(1,0, \cdots, 0)$.

Proof. Let $U$ be a neighborhood of the point $y$ such that $\bar{U} \subset \boldsymbol{R}_{+}^{n}$ 
and $\phi \in C_{0}^{\infty}\left(\boldsymbol{R}_{+}^{n}\right)$ be equal to 1 in $U$. Then we have

$$
\phi(x) P\left(D_{x}\right) E(x, y)=\delta(x-y) \text { in } \boldsymbol{R}^{n},
$$

so it follows from Leibniz's formula that

$$
P(D) f(x)=\delta(x-y)+g(x) \text { in } \boldsymbol{R}^{n},
$$

where $f(x)=\phi(x) E(x, y)$ and $g(x)=\sum_{|\alpha| \geq 1}\left(D^{\alpha} \phi(x)\right)\left(P^{(\alpha)}\left(D_{x}\right) E(x, y) / \alpha\right.$ ! are distributions with compact support. Taking Fourier-Laplace transforms we have

$$
P(\zeta) \widehat{f}(\zeta)=\exp \{-i y \cdot \zeta\}+\widehat{g}(\zeta), \zeta \in \boldsymbol{C}^{n}=\boldsymbol{\Xi}^{n}+i \boldsymbol{\Xi}^{n},
$$

where $\hat{f}(\zeta)=\mathscr{F} f, \widehat{g}(\zeta)=\mathscr{F} g$ are entire functions. If $P(\zeta)$ is a nonvanishing constant, the conditions (1.1) and (1.2) are satisfied, so we assume that $P(\zeta)$ is not a constant. Then $g$ cannot identically vanish because $P(\zeta)$ has zeros. Since $g \in \mathcal{E}^{\prime}\left(\boldsymbol{R}^{n}\right)$ we have for some constants $C$ and $N$

$$
|\widehat{g}(\zeta)| \leq C(1+|\zeta|)^{N} \exp h(\operatorname{Im} \zeta)
$$

where

$$
h(\eta)=\operatorname{Max} x \cdot \eta, \quad x \in \operatorname{supp} g, \quad \eta \in \boldsymbol{\Xi}^{n} .
$$

We note that

$$
h(-\vartheta)>0 .
$$

In fact, this follows from the fact that the support of $g$ is contained in (supp $\phi-U) \cap(K+y)$. From (2.2) and (2.3) we find for any $\zeta \in \mathbb{C}^{n}$ with $P(\zeta)=0$

$$
|\exp \{-i y \cdot \zeta\}| \leq C(1+|\zeta|)^{N} \exp h(\operatorname{Im} \zeta) .
$$

After taking logarithms of both sides, we get for any $\zeta \in \mathbb{C}^{n}$ with $P(\zeta)=0$

$$
-h(\operatorname{Im} \zeta)+y \cdot \operatorname{Im} \zeta \leq C_{1}+C_{2} \log (1+|\zeta|)
$$

where $C_{1}$ and $C_{2}$ are some positive constants independent of $\zeta$.

We shall now prove that the plane $x \cdot \vartheta=0$ is non-characteristic with respect to $P(D)$, i.e., $P^{0}(\vartheta) \neq 0$. We will argue by contradiction. Suppose that $P^{0}(\vartheta)=0$. Since $P^{0}(\xi) \not \equiv 0$, we take a $\xi^{0} \in \boldsymbol{\xi}^{n}$ such that 
$P^{0}\left(\xi^{0}\right) \neq 0$, and consider the polynomial in two variable $t$ and $s$ :

$$
P\left(s t\left(\xi^{0}-\vartheta\right)+t \vartheta\right)=P^{0}\left(s\left(\xi^{0}-\vartheta\right)+\vartheta\right) t^{m}+\cdots=c_{0}(s) t^{m}+\cdots .
$$

Since $c_{0}(0)=P^{0}(\vartheta)=0$ and $c_{0}(1)=P^{0}\left(\xi^{0}\right) \neq 0$, the algebraic equation in $t: P\left(s t\left(\xi^{0}-\vartheta\right)+t \vartheta\right)=0$ has a solution $t(s)$ in a neighborhood of $s=0$ such that

$$
t(s)=a s^{p}(1+o(1)), \quad s \rightarrow 0
$$

where $a$ is a non-zero complex number and $p$ is a negative rational number. In fact, the assumption $P^{0}(\vartheta)=0$ implies that $p$ is negative. If we choose the path $\gamma:|s| \leq \delta$ and $\arg s=(-\pi / 2-\arg a) / p$, we have

$$
\operatorname{Im} t(s)=-|a| \cdot|s|^{p}(1+o(1)) \quad \text { as } s \rightarrow 0 \text { along the path } \gamma
$$

and therefore

$$
\text { (2.8) } \operatorname{Im} \zeta(s)=\operatorname{Im}\left(s t(s)\left(\xi^{0}-\vartheta\right)+t(s) \vartheta\right)=-|a||s|^{p} \vartheta(1+o(1))
$$

as $s \rightarrow 0$ along the path $\gamma$, because $s t(s)=a s^{p+1}(1+o(1)), s \rightarrow 0$. Since $y \cdot \operatorname{Im} \zeta(s)=y^{\prime \prime} \cdot \operatorname{Im} \zeta^{\prime \prime}(s)=\operatorname{Im} s t(s)\left(\xi^{0}\right)^{\prime \prime} \cdot y^{\prime \prime}$, it follows from (2.5), (2.6) and (2.8) that

$$
|s|^{p} \leq C_{3}+C_{4} \log \left(1+|s|^{p}\right) \quad \text { as } s \rightarrow 0 \text { along the path } \gamma,
$$

where $C_{3}$ and $C_{4}$ are some positive constants. Then we have a contradiction. This proves that $P^{0}(\vartheta) \neq 0$.

Next we proceed to show (1.2) with $\vartheta=(1,0, \cdots, 0)$. Since $P^{0}(\vartheta)$ $\neq 0$, the algebraic equation $P(\xi+s \vartheta)=0$ in $s$ has $m$ roots $s_{j}(\xi), j=1, \cdots$, $m$, (counted according to multiplicity). Set

$$
\mu(r)=\sup _{\substack{|\xi|=r \\ j=1, \cdots, m}}-\operatorname{Im} s_{j}(\xi) .
$$

For every $s_{j}(\xi)$ we have from (2.6)

$$
h(-\vartheta) \operatorname{Im} s_{j}(\xi) \leq C_{1}+C_{2} \log \left(1+|\xi|+s_{j}(\xi)\right)
$$

because $y \cdot \vartheta=0$. Then we have

$$
\mu(r)=\operatorname{ar}^{p}(1+o(1)), \quad r \rightarrow+\infty,
$$

where $p$ is rational and $a$ is real. For we write

$$
P(\xi+s \vartheta)=R_{1}(\xi, \operatorname{Re} s, \operatorname{Im} s)+i R_{2}(\xi, \operatorname{Re} s, \operatorname{Im} s) .
$$


Here $R_{1}$ and $R_{2}$ are polynomials with constant coefficients in the $n+2$ real variables $\xi=\left(\xi_{1}, \cdots, \xi_{n}\right), \operatorname{Re} s$ and $\operatorname{Im} s$. If we set

$$
M_{r}=\left\{(\xi, \operatorname{Re} s, \operatorname{Im} s) ;|\xi|^{2}=r^{2}, R_{k}(\xi, \operatorname{Re} s, \operatorname{Im} s)=0, k=1,2\right\},
$$

then $\mu(r)=\sup _{(\xi, \operatorname{Re} s, \operatorname{Im} s) \in M_{r}}-\operatorname{Im} s$. By the Tarski-Seidenberg theorem we have either $(2.10)$ or $\mu(r) \equiv+\infty$ for all large $r$. Since $P^{0}(\vartheta) \neq 0$ as proved above, the roots $s_{j}(\xi)$ are multivalued continuous functions. Hence the function $\sup _{j=1, \cdots, m}\left|s_{j}(\xi)\right|$ is bounded when $\xi$ remains bounded. Thus $\mu(r)$ is finite for any finite $r \rightarrow 0$, so we have (2.10). From (2.9) and (2.10), it follows that

$$
-h(-\vartheta) \mu(r) \leq C_{1}+C_{2} \log \left(1+C_{3} r\right)
$$

where $C_{3}$ is a new positive constant. In view of (2.5) and (2.10) this inequality means that $\mu(r)$ remains bounded when $r \rightarrow+\infty$, namely there exists a real $\gamma_{0}$ such that

$$
-\operatorname{Im} s_{j}(\xi) \leq \gamma_{0} \text { for any } j(1 \leq j \leq m) \text { and for any } \xi \in \mathbb{\Xi}^{n} \text {. }
$$

Therefore, $P(\xi+s \vartheta) \neq 0$ when $\operatorname{Im} s<-\gamma_{0}$ and $\xi \in \mathbb{G}^{n}$. So the theorem is proved.

\section{$\S 3 . \quad$ Preliminaries for Latter Sections}

We denote by $\overline{\boldsymbol{R}_{+}^{n}}$ the space $\left\{x=\left(x^{\prime}, x_{n}\right) \in \boldsymbol{R}^{n} ; x_{n} \geq 0\right\}$, i.e., the closure of $\boldsymbol{R}_{+}^{n}$ and by $\partial \boldsymbol{R}_{+}^{n}$ the boundary plane $x_{n}=0$ of $\boldsymbol{R}_{+}^{n}$. Let $\boldsymbol{\Omega}$ be a non empty open set in $\boldsymbol{R}^{n}$ and let $\mathscr{D}(\Omega)$ denote the space of all complex $C^{\infty}$ functions with compact support in $\Omega$, which is equipped with the Schwartz topology. We set

$$
\dot{\mathscr{D}}\left(\overline{\boldsymbol{R}_{+}^{n}}\right)=\left\{\phi \in \mathscr{D}\left(\boldsymbol{R}^{n}\right) ; \operatorname{supp} \phi \subset \overline{\boldsymbol{R}_{+}^{n}}\right\} .
$$

This is a closed subspace of $\mathscr{D}\left(\boldsymbol{R}^{n}\right)$ and we give it the induced topology. We denote by $\mathscr{D}^{\prime}\left(\overline{\boldsymbol{R}_{+}^{n}}\right)$ the dual space of $\left.\mathscr{D}^{\left(\overline{\boldsymbol{R}_{+}^{n}}\right.}\right)$. By the Hahn-Banach theorem, any element of $\mathscr{D}^{\prime}\left(\overline{\boldsymbol{R}_{+}^{n}}\right)$ can be extended to a continuous linear form of $\mathscr{D}\left(\boldsymbol{R}^{n}\right)$, that is, a distribution on $\boldsymbol{R}^{n}$. On the other hand $\mathscr{D}\left(\boldsymbol{R}_{+}^{n}\right)$ is a dense subset of $\mathscr{D}\left(\overline{\boldsymbol{R}_{+}^{n}}\right)$ and therefore a continuous linear form on $\grave{\mathscr{D}}\left(\overline{\boldsymbol{R}_{+}^{n}}\right)$ is uniquely determined by its restriction to $\mathscr{D}\left(\boldsymbol{R}_{+}^{n}\right)$ and can be identified with an element of $\mathscr{D}^{\prime}\left(\boldsymbol{R}_{+}^{n}\right)$ consists of all distributions in $\boldsymbol{R}_{+}^{n}$. 
Thus the space $\mathscr{D}^{\prime}\left(\overline{\boldsymbol{R}_{+}^{n}}\right)$ consists of all distributions in $\boldsymbol{R}_{+}^{n}$ which are extendable to distributions on $\boldsymbol{R}^{n}$. Namely

$$
\mathscr{D}^{\prime}\left(\overline{\boldsymbol{R}_{+}^{n}}\right)=\mathscr{D}^{\prime}\left(\boldsymbol{R}^{n}\right) / \mathscr{D}^{\prime}\left(\overline{\boldsymbol{R}_{-}^{n}}\right),
$$

where $\dot{\mathscr{D}}^{\prime}\left(\overline{\boldsymbol{R}_{-}^{n}}\right)=\left\{u \in \mathscr{D}^{\prime}\left(\boldsymbol{R}^{n}\right)\right.$; supp $\left.u \subset \overline{\boldsymbol{R}_{-}^{n}}=\left\{x=\left(x^{\prime}, x_{n}\right) ; x_{n} \leq 0\right\}\right\}$. We denote by $C^{\infty}\left(I ; \mathscr{D}^{\prime}\left(\boldsymbol{R}^{n-1}\right)\right)$ the space of infinitely differentiable functions which are defined on interval $I$ in $\boldsymbol{R}^{1}$ with values in $\mathscr{D}^{\prime}\left(\boldsymbol{R}^{n-1}\right)$ equipped with the weak topology. When $I=[a, b), u \in C^{\infty}\left(I: \mathscr{D}^{\prime}\left(\boldsymbol{R}^{n-1}\right)\right)$ means by definition that for any $\phi \in \mathscr{D}\left(\boldsymbol{R}^{n-1}\right),\langle u(t), \phi\rangle$ is a $C^{\infty}$ function on $[a, b)$, that is, there exists a $C^{\infty}$ function $f_{\phi}(t)$ defined in $(a-\delta, b)$ for some $\delta>0$ such that $\langle u(t), \phi\rangle=f_{\phi}(t)$ on $[a, b)$. Then, for any $u \in C^{\infty}([a, b)$; $\left.\mathscr{D}^{\prime}\left(\boldsymbol{R}^{n-1}\right)\right)$ there exists a $v \in C^{\infty}\left(\left(a-\delta^{\prime}, b\right) ; \mathscr{D}^{\prime}\left(\boldsymbol{R}^{n-1}\right)\right)$ such that $u(t)=v(t)$ on $[a, b)$. This follows from a theorem due to Seeley [10] which guarantees the existence of a linear operator $T$ from $C^{\infty}([0, A))$ to $C^{\infty}((-A, A))(A>0)$ such that for any $f \in C^{\infty}([0, A)),(T f)(t)$ $=f(t)$ on $[0, A)$ and

$$
\operatorname{Max}_{t \in[-A, A]}\left|D_{t}^{k}(T f)(t)\right| \leq C_{k, A} \operatorname{Max}_{\substack{t \in[0, A] \\ 0 \leq j \leq k}}\left|D_{t}^{j} f(t)\right|,
$$

where $C_{k, A}$ is a positive constant depending on $k$ and $A$ but not on $f$. In fact, define a linear form $\langle v(t), \phi\rangle$ on $\mathscr{D}\left(\boldsymbol{R}^{n-1}\right)$ by $T\langle u(t), \phi\rangle$. Then it is easy to see that $v(t)$ is a $C^{\infty}$ function on $(-A, A)$ with values in $\mathscr{D}^{\prime}\left(\boldsymbol{R}^{n-1}\right)$. Clearly we have

$$
C^{\infty}\left([0, \infty), \mathscr{D}^{\prime}\left(R^{n-1}\right)\right) \subset \mathscr{D}^{\prime}\left(\overline{\boldsymbol{R}_{+}^{n}}\right) \subset \mathscr{D}^{\prime}\left(\boldsymbol{R}_{+}^{n}\right) .
$$

In our definition of a system $\left\{E(x, y) ; F_{j}(x), j=1, \cdots, q\right\}$ of fundamental solutions of the mixed problem (1.3)-(1.5), we assumed that $E(x, y) \in C^{\infty}\left(\left[0, y_{n}\right) ; \mathscr{D}^{\prime}\left(\boldsymbol{R}^{n-1}\right)\right) \cap C^{\infty}\left(\left(y_{n}, \infty\right) ; \mathscr{D}^{\prime}\left(\boldsymbol{R}^{n-1}\right)\right)$ and $F_{j}(x)$ $\in C^{\infty}\left([0, \infty) ; \mathscr{D}^{\prime}\left(\boldsymbol{R}^{n-1}\right)\right) .{ }^{4)} \quad$ These assumptions are reasonable because the fundamental solutions which we shall construct belong to such a space (see section 6). As already remarked, we do not assume that $\partial \boldsymbol{R}_{+}^{n}$ is non-characteristic for $P(D)$. However if $\partial \boldsymbol{R}_{+}^{n}$ is non-characteristic for $P(D)$, we need not assume that $E(x, y) \in C^{\infty}\left(\left[0, y_{n}\right) ; \mathscr{D}^{\prime}\left(\boldsymbol{R}^{n-1}\right)\right)$ $\cap C^{\infty}\left(\left(y_{n}, \infty\right) ; \mathscr{D}^{\prime}\left(\boldsymbol{R}^{n-1}\right)\right)$ and $F_{j}(x) \in C^{\infty}\left([0, \infty) ; \mathscr{D}^{\prime}\left(\boldsymbol{R}^{n-1}\right)\right)$. It is sufficient

4) See introduction. 
to assume that $E(x, y)$ and $F_{j}(x)$ belong to $\mathscr{D}^{\prime}\left(\overline{\boldsymbol{R}_{+}^{n}}\right)$, in view of the following.

Proposition 3. 1. Let $P(x, D)$ be a differential operator of order $m$ with coefficients in $C^{\infty}\left(\boldsymbol{R}^{n}\right)$ and assume that $\partial \boldsymbol{R}_{+}^{n}$ is noncharacteristic with respect to the differential operator. If $u \in \mathscr{D}^{\prime}\left(\overline{\boldsymbol{R}_{+}^{n}}\right)$ and $P(x, D) u \in C^{\infty}\left([0, \delta) ; \mathscr{D}^{\prime}\left(\boldsymbol{R}^{n-1}\right)\right)$, then we have that $u$ belongs to $C^{\infty}\left([0, \delta) ; \mathscr{D}^{\prime}\left(\boldsymbol{R}^{n-1}\right)\right)$.

Proof. Since $u \in \mathscr{D}^{\prime}\left(\overline{\boldsymbol{R}_{+}^{n}}\right)$, there exists a distribution $\widetilde{u} \in \mathscr{D}^{\prime}\left(\boldsymbol{R}^{n}\right)$ with $\widetilde{u}=u$ in $\boldsymbol{R}_{+}^{n}$. Let $\Omega$ be an open set of $\left\{x \in \boldsymbol{R}^{n} ; x_{n}<\delta\right\}$ such that the closure $\bar{\Omega}$ is compact. Then there exists a real $s$ such that $\phi \cdot \widetilde{u}$ $\in \mathcal{H}_{(s, 0)}\left(\boldsymbol{R}^{n}\right)$ for any $\phi \in C_{0}^{\infty}(\Omega)$, where $s$ is independent of $\phi$ and $\mathcal{H}_{(s, \sigma)}$ $=\left\{f \in \mathscr{S}^{\prime}\left(\boldsymbol{R}^{n}\right) ;\left(1+|\xi|^{2}\right)^{s / 2}\left(1+\left|\xi^{\prime}\right|^{2}\right)^{\sigma / 2} \widehat{f}(\xi) \in L^{2}\left(\boldsymbol{\Xi}^{n}\right)\right.$. If we set $v=\phi \widetilde{u}, g$ $=\phi P(x, D) \widetilde{u}$, Leibniz's formula gives

$$
P(x, D) v=g+Q(x, D) \widetilde{u} \text { in } \boldsymbol{R}^{n}
$$

where $Q(x, D)$ is a differential operator of order $m-1$ with coefficients in $C_{0}^{\infty}(\Omega)$ :

$$
Q(x, D) \widetilde{u}=\sum_{\substack{|\alpha|+\beta|\leq m\\| \alpha \mid>0}} b_{\alpha \beta}(x)\left(D^{\alpha} \phi\right)\left(D^{\beta} \widetilde{u}\right) .
$$

Let $\psi\left(x^{\prime}\right) \in C_{0}^{\infty}\left(\boldsymbol{R}^{n-1}\right)$. Then we get from (3.4)

$$
P(x, D)\left(\psi_{x^{\prime}}^{*}, v\right)=\psi_{x^{\prime}}^{*} g+\psi_{x^{\prime}}^{*}(Q(x, D) \widetilde{u})+\left[P(x, D), \psi_{x^{\prime}}^{*}\right] v,
$$

where $\left[P(x, D), \psi_{x^{\prime}}^{*}\right] v=P(x, D)\left(\psi_{x^{\prime}}^{*} v\right)-\psi_{x^{\prime}}^{*}(P(x, D) v)$. Since $\widehat{\psi}\left(\xi^{\prime}\right)$ is rapidly decreasing $C^{\infty}$ function of $\xi^{\prime}$, we have $\psi_{x^{\prime}}^{*}\left(\mathcal{H}_{(s, \sigma)}\right) \subset \mathcal{H}_{(s, \infty)}$ for any reals $s$ and $\sigma$. Thus $\psi_{x^{\prime}}^{*} v \in \mathcal{H}_{(s, \infty)}$ and so $\left.\psi_{x^{\prime}}^{*} v\right|_{\boldsymbol{R}_{+}^{n}}=\psi_{x^{\prime}}^{*}(\phi u) \in \mathcal{H}_{(s, \infty)}\left(\overline{\boldsymbol{R}_{+}^{n}}\right)$ $=\left\{w \in \mathscr{D}^{\prime}\left(\boldsymbol{R}_{+}^{n}\right)\right.$; There exists $\widetilde{w} \in \mathcal{H}_{(s, \infty)}$ such that $\widetilde{\boldsymbol{w}}=w$ in $\left.\boldsymbol{R}_{+}^{n}\right\}$. Here we denoted $\left.\phi\right|_{\boldsymbol{R}_{+}^{n}} u$ by $\phi u$ for simplicity. From the assumption on $P(x, D) u$ and the relation

$$
D^{\alpha}\left(g_{x^{\prime}}^{*} \psi\right)(x)=D_{n}^{\alpha_{n}}\left\langle g\left(y^{\prime}, x_{n}\right), D_{x^{\prime}}^{\alpha^{\prime}} \psi\left(x^{\prime}-y^{\prime}\right)\right\rangle,
$$

it follows that $\psi_{x^{\prime}}^{*} g \in C_{(o)}^{\infty}\left(\boldsymbol{R}_{+}^{n}\right)=\left\{\phi \in C^{\infty}\left(\boldsymbol{R}_{+}^{n}\right)\right.$; There exists $\widetilde{\phi} \in C_{0}^{\infty}\left(\boldsymbol{R}^{n}\right)$ such that $\tilde{\phi}=\phi$ in $\left.\boldsymbol{R}_{+}^{n}\right\}$. Consider the term $\phi_{x^{\prime}}^{*}(Q(x, D) u)$ in (3.5). Take $\chi \in C_{0}^{\infty}(\Omega)$ such that $\chi(x)=1$ on supp $\phi$. Since $\chi \widetilde{u} \in \mathcal{H}_{(s, 0)}$ and 
$Q(x, D)$ is of order $m-1$, we have $Q(x, D) \widetilde{u}=Q(x, D)(\chi \widetilde{u}) \in \mathcal{H}_{(s-m+1,0)}$. Hence $\psi_{x^{\prime}}^{*}(Q(x, D) u) \in \mathcal{H}_{(s-m+1, \infty)}\left(\overline{\boldsymbol{R}_{+}^{n}}\right)$. We have also $\left[P(x, D), \psi_{x^{\prime}}^{*}\right] \phi u$ $\in \mathcal{H}_{(s-m+1, \infty)}\left(\overline{\boldsymbol{R}_{+}^{n}}\right)$. In fact, we may assume from the assumption on $P(x, D)$ that $P(x, D)$ has the form

$$
P(x, D)=D_{n}^{m}+\sum_{\substack{|\alpha| \leq m \\ \alpha_{n}<m}} a_{\alpha}(x) D^{\alpha}=D_{n}^{m}+R(x, D) .
$$

Then $\left[P(x, D), \psi_{x^{\prime}}^{*}\right] v=\left[R(x, D), \psi_{x^{\prime}}^{*}\right] v$. Since the order of $R(x, D)$ with respect to $D_{n}$ is at most $m-1$, we have $R(x, D)\left(\psi_{x^{\prime}}^{*} v\right), \psi_{x^{\prime}}^{*}(R(x, D) v)$ $\in \mathcal{H}_{(s-m+1, \infty)}$ by using the fact the weight function $\left(1+|\xi|^{2}\right)^{\sigma / 2}$ is equivalent to $\left(1+\left|\xi^{\prime}\right|^{2}\right)^{\sigma / 2}\left(1+\left|\xi_{n}\right|^{2}\right)^{\sigma / 2}$ and so $\left[P(x, D), \psi_{x^{\prime}}^{*}\right] v \in \mathcal{H}_{(s-m+1, \infty)}$. This also follows from the fact that $\left[R(x, D), \psi_{x^{\prime}}^{*}\right]$ is a pseudo-differential operator whose symbol is

$$
-\sum_{\alpha} D_{x}^{\alpha} R(x, \xi)(\partial / \partial \xi)^{\alpha} \psi\left(\xi^{\prime}\right) / \alpha ! .
$$

Summing up, we have proved

$$
P(x, D)\left(\psi_{x^{\prime}}^{*}(\phi u)\right) \in \mathcal{H}_{(s-m+1, \infty)}\left(\overline{\boldsymbol{R}_{+}^{n}}\right) .
$$

By Theorem 4.3.1 in Hörmander [7], we get

$$
\psi_{x^{\prime}}^{*}(\phi u) \in \mathcal{H}_{(s+1, \infty)}\left(\overline{\boldsymbol{R}_{+}^{n}}\right) .
$$

Repeated application of the same argument gives that

$$
\psi_{x^{\prime}}^{*}(\phi u) \in \mathcal{H}_{(\infty, \infty)}\left(\overline{\boldsymbol{R}_{+}^{n}}\right) .
$$

Then

$$
\left(\psi_{x^{\prime}}^{*}(\phi u)\right)\left(0, x^{\prime}\right)=\left\langle(\phi u)\left(y^{\prime}, x_{n}\right), \breve{\psi}\left(y^{\prime}\right)\right\rangle_{y^{\prime}} \in C^{\infty}([0, \infty)) .
$$

Since $\phi \in C_{o}^{\infty}(\Omega)$ and $\phi \in C_{o}^{\infty}\left(\boldsymbol{R}^{n-1}\right)$ are arbitrary, we conclude that $u \in C^{\infty}\left([0, \delta) ; \mathscr{D}^{\prime}\left(\boldsymbol{R}^{n-1}\right)\right)$. This completes the proof.

Lemma 3. 2. Let $u\left(x_{n}\right)$ be a continuous function defined on the interval $[0, b]$ with values in $\mathscr{D}^{\prime}\left(\boldsymbol{R}_{x^{\prime}}^{n-1}\right)$. Then there exist constants $C, r$ and a compact set $K \subset \boldsymbol{R}^{n-1}$ such that

$$
\begin{gathered}
\left|\mathscr{F}_{x^{\prime}} u\left(x_{n}\right)\left(\zeta^{\prime}\right)\right| \leq C\left(1+\left|\zeta^{\prime}\right|\right)^{r} \exp h\left(\operatorname{Im} \zeta^{\prime}\right), \\
\zeta^{\prime}=\left(\zeta_{1}, \cdots, \zeta_{n-1}\right) \in C^{n-1}
\end{gathered}
$$


where $h\left(\operatorname{Im} \zeta^{\prime}\right)=\operatorname{Max}_{x^{\prime} \in K} x^{\prime} \cdot \operatorname{Im} \zeta^{\prime}$ and $C, r$ are independent of $x_{n} \in[0, b]$.

Proof. For any $\phi \in \mathcal{E}\left(\boldsymbol{R}_{x^{\prime}}^{n-1}\right)$ we have

$$
\sup _{x_{n} \in[0, b]}\left|\left\langle u\left(x_{n}\right), \phi\right\rangle\right|<\infty,
$$

that is, $\left\{u\left(x_{n}\right) ; x_{n} \in[0, b]\right\}$ is a weakly bounded set in $\mathcal{E}^{\prime}\left(R_{x^{\prime}}^{n-1}\right)$. Hence $\left\{u\left(x_{n}\right) ; x_{n} \in[0, b]\right\}$ forms a strongly bounded set in $\mathcal{E}^{\prime}\left(\boldsymbol{R}_{x^{\prime}}^{n-1}\right)$ and so there exists a compact subset $K$ of $\boldsymbol{R}_{x^{\prime}}^{n-1}$, a positive constant $C$ and an integer $r \geq 0$ such that $\bigcup_{x_{n} \in[0, b]} \operatorname{supp} u\left(x_{n}\right) \subset K$ and that

$$
\sup _{x_{n} \in[0, b]}\left|\left\langle u\left(x_{n}\right), \phi\right\rangle\right| \leq C \cdot \sup _{\substack{x^{\prime} \in K \\|\alpha| \leq r}}\left|D^{\alpha} \phi\left(x^{\prime}\right)\right|, \quad \phi \in \mathcal{E}\left(R_{x^{\prime}}^{n-1}\right),
$$

where $C$ is independent of $\phi$. On the other hand, we have

$$
\left(\mathscr{F}_{x^{\prime}} u\left(x_{n}\right)\right)\left(\zeta^{\prime}\right)=\left\langle u\left(x_{n}\right), \exp i x^{\prime} \cdot \zeta^{\prime}\right\rangle .
$$

Applying (3.7) to this relation, we get (3.6).

Q.E.D.

Now we present a basic lemma concerning a representation of solutions of equations which will be used in Sections 4 and 5 (see Hörmander [7]).

Let $P\left(D_{t}\right)=D_{t}^{m}+a_{m-1} D_{t}^{m-1}+\cdots+a_{0}$, where $D_{t}=-i d / d t$, be an ordinary differential operator with constant coefficients of order $m$. Assume that the zeros of the equation $P(\tau)=0$ are all non-real. Then let us denote them by $\tau_{1}^{+}, \cdots, \tau_{p}^{+}, \tau_{1}^{-}, \cdots, \tau_{m-p}^{-}$, where $\operatorname{Im} \tau_{j}^{+}>0, j=1, \cdots, p$ and $\operatorname{Im} \tau_{j}^{-}<0, j=1, \cdots, m-p$. Assume that $p \geq 1$. Let $B_{j}\left(D_{t}\right), j=1, \cdots, p$, be some other ordinary differential operators. Put

$$
P_{+}(\tau)=\Pi_{j=1}^{p}\left(\tau-\tau_{j}^{+}\right) .
$$

We define the Lopatinski determinant of the system $\left\{P\left(D_{t}\right), B_{j}\left(D_{t}\right)\right.$, $j=1, \cdots, p\}$ by

$$
R=\operatorname{det}\left((2 \pi i)^{-1} \int_{r} B_{j}(\tau) \tau^{k-1} P_{+}(\tau)^{-1} d \tau\right)_{j, k=1, \cdots, p},
$$

where $\gamma$ is a positively oriented contour in the complex $\tau$-plane enclosing the zeroes $\tau_{1}^{+}, \cdots, \tau_{p}^{+}$. We have

Lemma 3. 3. Assume that $R \neq 0$. If $f \in C_{(o)}^{\infty}\left(\overline{\boldsymbol{R}_{+}^{1}}\right), g_{j}, j=1, \cdots, p$, 
are complex numbers and $u$ is a solution of the inhomogeneous boundary problem:

$$
P\left(D_{t}\right) u=f, \quad t>0,\left.\quad B_{j}\left(D_{t}\right) u\right|_{t=0}=g_{j}, \quad j=1, \cdots, p,
$$

which belongs to $C_{(o)}^{\infty}\left(\overline{\boldsymbol{R}_{+}^{1}}\right)$, then it follows $u=v_{1}+v_{2}$, where

$$
\begin{gathered}
v_{1}=(2 \pi)^{-1} \int \exp \{i t \tau\} \mathscr{L}\left[f_{0}\right](\tau) P(\tau)^{-1} d \tau, \\
v_{2}=(2 \pi i)^{-1} \int \sum_{j, k=1}^{p} \exp \{i t \tau\} \tau^{k-1} R_{j k}\left(g_{j}-\left.B_{j}\left(D_{t}\right) v_{1}\right|_{t=0}\right)\left(P_{+}(\tau) R\right)^{-1} d \tau .
\end{gathered}
$$

Here $f_{0}$ denotes a compactly supported $C^{\infty}$ extension of $f$ to $t<0,{ }^{5)}$ $\mathscr{F}\left[f_{0}\right]$ denotes the Fourier transform of $f_{0}$, and $R_{j k}$ denote the $(j, k)$ cofactor of the matrix $\left((2 \pi i)^{-1} \int B_{j}(\tau) \tau^{k-1}\left(P_{+}(\tau)\right)^{-1} d \tau\right)_{j, k=1, \ldots, p}$.

\section{§4. Hyperbolicity of the Lopatinski Determinant}

Let $P(\xi)$ be a hyperbolic polynomial of order $m$ with respect to $\vartheta$. Then it follows that the principal part $P^{0}(\xi)$ is hyperbolic with respect to $\vartheta$ and that a homogeneous hyperbolic polynomial has only real characteristics. The component $\Gamma=\Gamma(P)=\Gamma(P, \vartheta)$ of the set $\boldsymbol{E}^{n}-\left\{\xi \in \boldsymbol{\Xi}^{n} ; P^{0}(\xi)=0\right\}$ which contains $\vartheta$ is an open convex cone with its vertex at the origin and we have

(4.1) $P(\xi+i \eta) \neq 0$ when $\xi \in \boldsymbol{E}^{n}$ and $\eta \in-\Gamma-s$ with $s$ large enough.

The localization $P_{\xi^{\circ}}(\xi)$ of $P(\xi)$ at $\xi^{0}$ is defined by

$$
\tau^{m} P\left(\tau^{-1} \xi^{0}+\xi\right)=\tau^{m} \xi^{0} P_{\xi 0}(\xi)+O\left(\tau^{m \xi^{0}+1}\right) \text { as } \tau \rightarrow 0,
$$

where $P_{\xi^{\circ}}(\xi)$ is the first coefficients that does not vanish identically in $\xi$. Then it follows that the localization $P_{\xi 0}(\xi)$ of $P(\xi)$ at any real point $\hat{\xi}^{0}$ is hyperbolic with respect to $\vartheta$ and that

$$
\Gamma=\Gamma(P, \vartheta) \subset \Gamma_{\xi^{0}}=\Gamma\left(P_{\xi^{0}}, \vartheta\right),
$$

For further details we refer to Atiyah, Bott and Gåding [1].

Now we write the polynomial $P(\xi)$ in terms of power of $\xi_{n}$.

$$
P(\xi)=P\left(\xi^{\prime}, \xi_{n}\right)=\sum_{j=1}^{m^{\prime}} q_{j}\left(\xi^{\prime}\right) \xi_{n}^{m^{\prime}-j},
$$

5) See Seeley [10]. 
where $q_{0}\left(\xi^{\prime}\right)$ is not identically 0 . So $m^{\prime} \leq m$, in particular $m=m^{\prime}$ if the hyperplane $x_{n}=0\left(: \partial \boldsymbol{R}_{+}^{n}\right)$ is non-characteristic for $P(\xi)$. Then we see that $q_{0}\left(\xi^{\prime}\right)$ is the localization of $P(\xi)$ at $N=(0, \cdots, 0,1)$. Hence we have

$$
q_{0}\left(\xi^{\prime}+i \eta^{\prime}\right) \neq 0 \text { when } \xi^{\prime} \in \boldsymbol{\Xi}^{n-1} \text { and } \eta \in-\Gamma_{\xi^{0}}-s \vartheta \text {, }
$$

in particular $\eta \in-\Gamma-s \vartheta$ with $s$ large enough (Note the relation (4.3)). ${ }^{6)}$ If we set

$$
\Gamma_{0}=\left\{\eta^{\prime} \in \mathbb{G}^{n-1} ;\left(\eta^{\prime}, 0\right) \in \Gamma\right\}=\Xi_{\eta^{\prime}}^{n-1} \cap \Gamma,
$$

it follows from (4.1) that the roots of the equation $P\left(\zeta^{\prime}, \lambda\right)=0$ in $\lambda$ are never real when $\zeta^{\prime}=\xi^{\prime}+i \eta^{\prime} \in \boldsymbol{\xi}^{n-1}-i \Gamma_{0}-i s \vartheta^{\prime}$ with $s$ large enough. By (4.4) the roots of $P\left(\zeta^{\prime}, \lambda\right)=0$ in $\lambda$ are (multivalued) continuous functions of $\zeta^{\prime} \in \boldsymbol{\Xi}^{n-1}-i \Gamma_{0}-i s \vartheta^{\prime}$ and therefore the number of roots with positive imaginary part, counted according to multiplicity, is constant when $\zeta^{\prime} \in \Xi^{n-1}-i \Gamma_{0}-i s \vartheta^{\prime}$. The roots of $P\left(\zeta^{\prime}, \lambda\right)=0$ in $\lambda$ with positive imaginary part we denote by $\lambda_{1}^{+}\left(\zeta^{\prime}\right), \cdots, \lambda_{p}^{+}\left(\zeta^{\prime}\right)$ and those with negative imaginary part by $\lambda_{p+1}^{-}\left(\zeta^{\prime}\right), \cdots, \lambda_{m^{\prime}}^{-}\left(\zeta^{\prime}\right)$. Put

$$
P_{+}\left(\zeta^{\prime}, \lambda\right)=\Pi_{j=1}^{p}\left(\lambda-\lambda_{j}^{+}\left(\zeta^{\prime}\right)\right), \quad \zeta^{\prime} \in \boldsymbol{\Xi}^{n-1}-i \Gamma_{0}-i s \vartheta^{\prime} .
$$

In this section we consider the case where the number of boundary conditions $B_{j}(D)$ equals exactly $p$. In particular, if $p=0$, boundary conditions are not imposed. Therefore, we assume that $p \geq 1$ in this section. We define the Lopatinski determinant of the system $\left\{P(D) ; B_{j}(D)\right.$, $j=1, \cdots, p\}$ by

$$
R\left(\zeta^{\prime}\right)=\operatorname{det}\left((2 \pi i)^{-1} \int_{r\left(\zeta^{\prime}\right)} B_{j}\left(\zeta^{\prime}, \lambda\right) \lambda^{k-1} P_{+}\left(\zeta^{\prime}, \lambda\right)^{-1} d \lambda\right)_{j, k=1, \ldots, p}
$$

$\zeta^{\prime} \in \mathbb{G}^{n-1}-i \Gamma_{0}-i s \vartheta^{\prime}$ with $s$ large enough, where $\gamma\left(\zeta^{\prime}\right)$ is a positively oriented contour in the complex $\lambda$-plane enclosing the roots $\lambda_{1}^{+}\left(\zeta^{\prime}\right), \cdots$, $\lambda_{p}^{+}\left(\zeta^{\prime}\right)$. Denote by $\dot{\Gamma}$ the projection of $\Gamma$ in $\boldsymbol{\Xi}^{n-1}$, that is,

$$
\dot{\Gamma}=\left\{\xi^{\prime} \in \boldsymbol{G}^{n-1} ;\left(\xi^{\prime}, \xi_{n}\right) \in \Gamma \text { for some } \xi_{n} \in \boldsymbol{G}\right\},
$$

which coincides with one defined in Sakamoto [9]. The following two

6) These fact are pointed out by $K$. Kasahara. He also showed that his discussions in [8] are valid in this case (unpublished). 
lemmas are due to Sakamoto [9].

Lemma 4. 1 ([9]). $R\left(\zeta^{\prime}\right)$ is holomorphic in $\boldsymbol{\Xi}^{n}-i \Gamma-i s \vartheta^{\prime}$ with s large enough.

Lemma 4. 2 ([9]). Let $t$ be a complex parameter. Then we have

$$
R\left(t \zeta^{\prime}\right)=t^{h_{0}}\left\{R^{0}\left(\zeta^{\prime}\right)+t^{-1} R^{1}\left(\zeta^{\prime}\right)+t^{-2} R^{2}\left(\zeta^{\prime}\right)+\cdots\right\},
$$

where the convergence is uniform in $K \times\left\{\lambda \in C ;|\lambda|>T_{K}\right\}$ for every compact set $K$ in $\boldsymbol{\Xi}^{n-1}-i \dot{\Gamma}$ and for some $T_{K}>0$ depending on $K$. Furthermore

(I) $R^{0}\left(\zeta^{\prime}\right)$ does not vanish identically in $\zeta^{\prime}$ and $h_{0}$ is an integer.

(II) every $R^{j}\left(\zeta^{\prime}\right)$ is holomorphic in $\dot{\Gamma}=\bigcup_{z \in C-\{0\}} z\left(\boldsymbol{\Xi}^{n-1}-i \dot{\Gamma}\right)$,

(III) $\quad R^{j}\left(t \zeta^{\prime}\right)=t^{h_{0}-j} R^{j}\left(\zeta^{\prime}\right), \zeta^{\prime} \in \dot{\Gamma}, t \in C-\{0\}$.

Main purpose of this section is to show the following.

Proposition 4. 3. Assume that $P(D)$ is hyperbolic with respect to $\vartheta$ and that $p$ which is the number of the roots with positive imaginary part of the equation $P\left(\xi^{\prime}-i s \vartheta^{\prime}, \lambda\right)=0$ in $\lambda$ for large positive $s$ is greater than 0 . If in the mixed problem (1.3)-(1.5) the number of boundary conditions is equal to $p$ and there exist distributions on $\boldsymbol{R}_{+}^{n}, F_{j}(x), j=1, \cdots, p$, which satisfy the equations (1. 10) and (1.11) and belong to $C^{\infty}\left([0,0) ; \mathscr{D}^{\prime}\left(\boldsymbol{R}^{n-1}\right)\right)$ for some positive number $\delta$, rehose support is contained in $K \cap \overline{\boldsymbol{R}_{+}^{n}}$, then the Lopatinski determinant of the system $\left\{P(D) ; B_{j}(D), j=1, \cdots, p\right\}$ is hyperbolic with respect to $\vartheta^{\prime}$, that is,

(I) $R^{0}\left(\vartheta^{\prime}\right) \neq 0$,

(II) there exists a real number $\gamma_{1}$ such that

$$
R\left(\xi^{\prime}+s \vartheta^{\prime}\right) \neq 0 \text { when } \xi^{\prime} \in \boldsymbol{\Xi}^{n-1} \text { and } \operatorname{Im} s<\gamma_{1} \text {. }
$$

Proof. Let $U$ and $U^{\prime}$ be open neighborhoods of the origin in $\boldsymbol{R}^{n}$ such that the closure of $U$ is contained in $U^{\prime}$, that the closure of $U^{\prime}$ is contained in $\boldsymbol{R}^{n-1} \times(-\delta / 2, \delta / 2)$ and compact in $\boldsymbol{R}^{n}$. Choose 
$\phi \in C_{0}^{\infty}\left(U^{\prime} \times(-\delta / 2, \delta / 2)\right)$ with $\phi(x)=1$ in $U$. Then

$$
P(D)\left(\phi F_{k}\right)(x)=f_{k}(x), \quad x \in \boldsymbol{R}_{+}^{n} .
$$

$$
\left.B_{j}(D)\left(\phi F_{k}\right)(x)\right|_{x_{n}=0}=\delta_{j k} \delta\left(x^{\prime}\right)+g_{j k}\left(x^{\prime}\right), \quad x^{\prime} \in \boldsymbol{R}^{n-1},
$$

where $f_{k}=P(D)(\phi-1) F_{k}$ and $g_{j k}=\left.B_{j}(D)(\phi-1) F_{k}\right|_{x_{n}=0}$ are distributions with compact support. Taking partial Fourier-Laplace transforms of both sides of (4.10) and (4.11), we obtain

$$
\begin{aligned}
& P\left(\zeta^{\prime}, D_{n}\right)\left(\widehat{\phi F}_{k}\right)\left(\zeta^{\prime}, x_{n}\right)=\widehat{f}_{k}\left(\zeta^{\prime}, x_{n}\right), x_{n}>0, \\
& \left.B_{j}\left(\zeta^{\prime}, D_{n}\right)\left(\widehat{\phi F}_{k}\right)\left(\zeta^{\prime}, x_{n}\right)\right|_{x_{n}=0}=\delta_{j k}+\widehat{g}_{j k}\left(\zeta^{\prime}\right) .
\end{aligned}
$$

Now we apply the Euclidean algorithm to the polynomials in $\lambda: B_{j}\left(\zeta^{\prime}, \lambda\right)$, $P\left(\zeta^{\prime}, \lambda\right)$ and $P_{+}\left(\zeta^{\prime}, \lambda\right)$. Then we have

(4. 14) $B_{j}\left(\zeta^{\prime}, \lambda\right)=Q_{j}\left(\zeta^{\prime}, \lambda\right) P\left(\zeta^{\prime}, \lambda\right)+T_{j}\left(\zeta^{\prime}, \lambda\right) P_{+}\left(\zeta^{\prime}, \lambda\right)+B_{j}^{*}\left(\zeta^{\prime}, \lambda\right)$, where $Q_{j}\left(\zeta^{\prime}, \lambda\right), T_{j}\left(\zeta^{\prime}, \lambda\right)$ and $B_{j}^{*}\left(\zeta^{\prime}, \lambda\right)$ are polynomials in $\lambda$ whose coefficients are holomorphic functions of $\zeta^{\prime}$ in $\boldsymbol{\Xi}^{n-1}-i \gamma_{0} \vartheta^{\prime}-i \dot{\Gamma}$ and of polynomial growth in $\zeta^{\prime}$. Furthermore, $\operatorname{deg}_{\lambda} Q_{j}<r_{j}-m^{\prime}, \underset{\lambda}{\operatorname{deg}} T_{j}<m^{\prime}-p$ and $\operatorname{deg} B_{j}^{*}<p$. Here $r_{j}$ is the degree of $B_{j}\left(\zeta^{\prime}, \lambda\right)$ in $\lambda$. Substituting (4.14) in (4.13), we find

$$
\begin{aligned}
\left.B_{j}\left(\zeta^{\prime}, D_{n}\right)\left(\widehat{\phi F}_{k}\right)\left(\zeta^{\prime}, x_{n}\right)\right|_{x_{n}=0} \\
=\delta_{j k}+\widehat{g}_{j k}\left(\zeta^{\prime}\right)-\left.Q_{j}\left(\zeta^{\prime}, D_{n}\right) \widehat{f}_{k}\left(\zeta^{\prime}, x_{n}\right)\right|_{x_{n}=0} \\
\quad-\left.T_{j}\left(\zeta^{\prime}, D_{n}\right) P_{+}\left(\zeta^{\prime}, D_{n}\right)\left(\widehat{\phi F}_{k}\right)\left(\zeta^{\prime}, x_{n}\right)\right|_{x_{n}=0}
\end{aligned}
$$

We write $B_{j}^{*}\left(\zeta^{\prime}, D_{n}\right)$ in terms of porvers of $D_{n}$;

$$
B_{j}^{*}\left(\zeta^{\prime}, D_{n}\right)=\sum_{k=1}^{p} b_{j k}\left(\zeta^{\prime}\right) D_{n}^{p-k}
$$

where all $b_{j k}\left(\zeta^{\prime}\right)$ are holomorphic in $\boldsymbol{\Xi}^{n-1}-i \gamma_{0} \vartheta^{\prime}-i \dot{\Gamma}$. Since

$$
\begin{gathered}
\frac{1}{2 \pi i} \int_{\tau\left(\zeta^{\prime}\right)} \lambda^{j} P_{+}\left(\zeta^{\prime}, \lambda\right)^{-1} d \lambda= \begin{cases}0 & \text { for } j=0,1, \cdots, p-2, \\
1 & \text { for } j=p-1,\end{cases} \\
\zeta^{\prime} \in \boldsymbol{\Xi}^{n-1}-i \gamma_{0} \vartheta^{\prime}-i \dot{\Gamma},
\end{gathered}
$$

we have

$$
R\left(\zeta^{\prime}\right)=\operatorname{det}\left(b_{j k}\left(\zeta^{\prime}\right)\right)_{j, k=1, \cdots, p}, \zeta^{\prime} \in \boldsymbol{E}^{n-1}-i \gamma_{0} \vartheta^{\prime}-i \dot{\Gamma} .
$$

From (4.15) and (4.18), we obtain 


$$
\begin{array}{r}
R\left(\zeta^{\prime}\right) \operatorname{det}\left(\left.D_{n}^{j-1} F_{k}\left(\zeta^{\prime}, x_{n}\right)\right|_{x_{n}=0}\right)_{j, k=1, \cdots, p} \\
=1+S\left(\zeta^{\prime}\right), \zeta^{\prime} \in \boldsymbol{\Xi}^{n-1}-i \gamma_{0} \vartheta^{\prime}-i \dot{\Gamma} .
\end{array}
$$

We first consider the case where $S\left(\zeta^{\prime}\right) \equiv 0$ in $\Xi^{n-1}-i \gamma_{0} \vartheta^{\prime}-i \dot{\Gamma}$. Then $R\left(\zeta^{\prime}\right) \neq 0$ in $\boldsymbol{\Xi}^{n-1}-i \gamma_{0} \vartheta^{\prime}-\dot{\Gamma}$. Suppose that $R^{0}(\vartheta)=0$. Since $R\left(\tau \vartheta^{\prime}\right)$ $=\tau^{h_{0}}\left(R^{0}\left(\vartheta^{\prime}\right)+\tau^{-1} R^{1}\left(\vartheta^{\prime}\right)+\cdots\right) \neq 0$ for any $\tau \in C^{1}$ with $|\tau|$ large enough, there exists an integer $j_{0} \geq 1$ such that $R^{j}\left(\vartheta^{\prime}\right)=0, j=0, \cdots, j_{0}-1$ and $R^{j_{0}}\left(\vartheta^{\prime}\right) \neq 0$. Since $R^{0}\left(\zeta^{\prime}\right) \not \equiv 0$, take a $\zeta^{0 \prime} \in \boldsymbol{\Xi}^{n-1}-i \dot{\Gamma}$ such that $R^{0}\left(\zeta^{0 \prime}\right) \neq 0$ and consider the function of $(s, z)$ :

(4. 20) $f(s, z)=R^{0}\left(s\left(\zeta^{0 \prime}-\vartheta^{\prime}\right)+\vartheta^{\prime}\right)+z R^{1}\left(s\left(\zeta^{\prime \prime}-\vartheta^{\prime}\right)+\vartheta^{\prime}\right)+\cdots$.

This function is holomorphic in a neighborhood of the origin of $C^{\mathbf{2}}$. Since by assumption $f(0,0)=\cdots=(\partial / \partial z)^{j_{0}-1} f(0,0)=0$ and $(\partial / \partial z)^{j_{0}}$ $\times f(0,0) \neq 0$, we can write by applying the Weierstrass preparation theorem (see, for example, [4]) to $f$

$$
f(s, z)=\left(z^{j_{0}}+\sum_{j=1}^{j_{0}} a_{j}(s) z^{j_{0}-j}\right) u(s, z),
$$

where $u(s, z)$ is holomorphic and $u(s, z) \neq 0$ in a neighborhood of $(s, z)$ $=(0,0)$ and $a_{j}(0)=0, j=1, \cdots, j_{0}$. Then the equation $f(s, z)=0$ has a solution $z(s) \not \equiv 0$ which is an analytic function of $s^{1 / \rho}$ in a neighborhood of the origin and vanishes at the origin:

$$
z(s)=\sum_{j=1} c_{j}\left(s^{1 / \rho}\right)^{j} \quad|s|<d,
$$

where $d$ is a positive rational number. Put

$$
t(s)=1 / z(s)
$$

Then we have

$$
t(s)=a s^{\nu}(1+o(1)), \quad \text { as } s \rightarrow 0
$$

where $\nu$ is a negative rational number and $a$ is a complex number. If we choose the path: $0<|s|<d$ and $\arg \left(a s^{\nu}\right)=-\pi / 2$, we have

$$
t(s) s\left(\zeta^{\prime \prime}-\vartheta^{\prime}\right)+t(s) \vartheta^{\prime} \in \Xi^{n-1}-i \gamma_{0} \vartheta^{\prime}-i \dot{\Gamma}
$$

and

$$
R\left(t(s)\left(\zeta^{\prime \prime}-\vartheta^{\prime}\right)+t(s) \vartheta^{\prime}\right)=0
$$

by taking $d>0$ sufficiently small if necessary. This contradicts that 
$R\left(\zeta^{\prime}\right) \neq 0$ in $\Xi^{n-1}-i \gamma_{0} \vartheta^{\prime}-i \dot{\Gamma}$. Then we have $R^{0}\left(\vartheta^{\prime}\right) \neq 0$ when $S\left(\zeta^{\prime}\right) \equiv 0$.

Next we consider the case where $S\left(\zeta^{\prime}\right)$ does not vanish identically in $\Xi^{n-1}-i \gamma_{0} \vartheta^{\prime}-i \dot{\Gamma}$. Put

$$
H=\left\{x^{\prime} ; x=\left(x^{\prime}, x_{n}\right) \in(\operatorname{supp} \phi-U) \cap K \text { for sonie } x_{n} \geq 0\right\} \subset \boldsymbol{R}^{n-1} .
$$

Then $\operatorname{supp}_{x^{\prime}} f_{k} \subset H$ and supp $g_{j k} \subset H$. Since the compact set $H$ does not contains the origin in $\boldsymbol{R}^{n-1}$ and $x_{1}=x^{\prime} \cdot \vartheta^{\prime}>0$ on $H$, we have

$$
h\left(-\vartheta^{\prime}\right)=\operatorname{Max}_{x^{\prime} \in H} x^{\prime} \cdot\left(-\vartheta^{\prime}\right)<0 .
$$

Further we have for some constants $C$ and $N$

$$
\left|\widehat{g}_{j k}\left(\zeta^{\prime}\right)\right| \leq C\left(1+\left|\zeta^{\prime}\right|\right)^{N} \exp h\left(\operatorname{Im} \zeta^{\prime}\right)
$$

and

$$
\text { (4. 27) }\left|Q_{j}\left(\zeta^{\prime}, D_{n}\right) \widehat{f}_{k}\left(\zeta^{\prime}, x_{n}\right)\right|_{x_{n}=0} \mid \leq C\left(1+\left|\zeta^{\prime}\right|\right)^{N} \exp h\left(\operatorname{Im} \zeta^{\prime}\right) \text {. }
$$

We shall also denote by $C$ and $N$ some inessential constants which occur in later estimates. Now we estimate the term $T_{j}\left(\zeta^{\prime}, D_{n}\right) P_{+}\left(\zeta^{\prime}, D_{n}\right)$ $\times\left.\left(\widehat{\phi F}_{k}\right)\left(\zeta^{\prime}, x_{n}\right)\right|_{x_{n}=0}$ in (4.15). For simplicity we put $v_{k}\left(\zeta^{\prime}, x_{n}\right)$ $=P_{+}\left(\zeta^{\prime}, D_{n}\right)\left(\widehat{\phi F}_{k}\right)\left(\zeta^{\prime}, x_{n}\right)$. Then $\operatorname{supp}_{x_{n}} v_{k}\left(\zeta^{\prime}, x_{n}\right) \subset[0, \delta / 2]$ and $v_{k}\left(\zeta^{\prime}, x_{n}\right)$ is a solution of the equation

$$
P_{-}\left(\zeta^{\prime}, D_{n}\right) v_{k}\left(\zeta^{\prime}, x_{n}\right)=\widehat{f}_{k}\left(\zeta^{\prime}, x_{n}\right), x_{n}>0,
$$

where $P_{-}\left(\zeta^{\prime}, D_{n}\right)=P\left(\zeta^{\prime}, D_{n}\right) / P_{+}\left(\zeta^{\prime}, D_{n}\right)$ is a polynomial in $D_{n}$ whose coefficients are holomorphic function of $\zeta^{\prime}$ in $\Xi^{n-1}-i \gamma_{0} \vartheta^{\prime}-i \dot{\Gamma}$. Furthermore these functions are of polynomial growth in $\zeta^{\prime}$. We define $\widehat{f}_{k}\left(\zeta^{\prime}, x_{n}\right)=0$ for $x_{n}<0$. Since the equation $P_{-}(\zeta, \lambda)=0$ in $\lambda$ has only roots $\lambda\left(\zeta^{\prime}\right)$ with $\operatorname{Im} \lambda\left(\zeta^{\prime}\right)<0$ when $\zeta^{\prime} \in \boldsymbol{E}^{n-1}-i \gamma_{0} \vartheta^{\prime}-i \dot{\Gamma}$, we have

$$
\begin{gathered}
v_{k}\left(\zeta^{\prime}, x_{n}\right)=(2 \pi)^{-1} \int_{-\infty}^{+\infty} \exp \left\{i \lambda x_{n}\right\} \mathscr{F}_{x_{n}}\left[\widehat{f}_{k}\left(\zeta^{\prime}, x_{n}\right)\right](\lambda) P_{-}\left(\zeta^{\prime}, \lambda\right) d \lambda, \\
x_{n} \geq 0 .
\end{gathered}
$$

Hence we have

$$
\begin{aligned}
& \left|D_{n}^{l} v_{k}\left(\zeta^{\prime}, 0\right)\right| \\
& \leq \leq \\
& \quad \times\left\{\int_{-\infty}^{+\infty}\left|\mathscr{F}_{x_{n}}\left[\widehat{f}_{k}\left(\zeta^{\prime}, x_{n}\right)\right](\lambda)\right|^{2} d \lambda\right\}^{1 / 2} \\
& \quad \times\left\{\int_{-\infty}^{+\infty}\left|\lambda^{l}\left(P_{-}\left(\zeta^{\prime}, \lambda\right)\right)^{-1}\right|^{2} d \lambda\right\}^{1 / 2}
\end{aligned}
$$




$$
\leq C\left\{\int_{0}^{+\infty}\left|\widehat{f}_{k}\left(\zeta^{\prime}, x_{n}\right)\right|^{2} d x_{n}\right\}^{1 / 2}, \quad \text { if } \quad 0 \leq l<m^{\prime}-p
$$

Now, we know that

$$
\left|\widehat{f}_{k}\left(\zeta^{\prime}, x_{n}\right)\right| \leq C\left(1+\left|\zeta^{\prime}\right|\right)^{N} \exp h\left(\operatorname{Im} \zeta^{\prime}\right)
$$

where the constants $C$ and $N$ are independent of $x_{n} \geq 0$. Since $\operatorname{deg} T_{j}\left(\zeta^{\prime}, \lambda\right)<m^{\prime}-p$, we obtain from (4.28)

$$
\begin{aligned}
\mid T_{j}\left(\zeta^{\prime}, D_{n}\right) P_{+}\left(\zeta^{\prime}, D_{n}\right) \widehat{\left.\left(\phi F_{k}\right)\left(\zeta^{\prime}, x_{n}\right)\right|_{x_{n}=0} \mid} \\
\quad=\left|T_{j}\left(\zeta^{\prime}, D_{n}\right) v_{k}\left(\zeta^{\prime}, x_{n}\right)\right|_{x_{n}=0} \mid \\
\leq C\left(1+\left|\zeta^{\prime}\right|^{N} \exp \left\{h\left(\operatorname{Im} \zeta^{\prime}\right)\right\} .\right.
\end{aligned}
$$

From (4.15), (4.18), (4.19), (4.26), (4.27) and (4.29), we deduce that

$$
\left|S\left(\zeta^{\prime}\right)\right| \leq C\left(1+\left|\zeta^{\prime}\right|\right)^{N} \exp \left\{r h\left(\operatorname{Im} \zeta^{\prime}\right)\right\}
$$

where $r$ is a positive integer. Then (4.17) implies that

$$
1 \leq C\left(1+\left|\zeta^{\prime}\right|\right)^{N} \exp \left\{r h\left(\operatorname{Im} \zeta^{\prime}\right)\right\} \quad \text { for any } \zeta^{\prime} \in \boldsymbol{\Xi}^{n-1}-i \gamma_{0} \vartheta^{\prime}-i \dot{\Gamma}
$$

such that $R\left(\zeta^{\prime}\right)=0$. Hence we have

(4. 30) $-h\left(\operatorname{Im} \zeta^{\prime}\right) \leq C_{1}+C_{2} \log \left(1+\left|\zeta^{\prime}\right|\right)$ for any $\zeta^{\prime}$ with $R\left(\zeta^{\prime}\right)=0$.

Here $C_{1}$ and $C_{2}$ are some positive constants independent of $\zeta^{\prime}$.

Now let us show $R^{0}\left(\vartheta^{\prime}\right) \neq 0$. If $R^{j}\left(\vartheta^{\prime}\right)=0$ for all $j=0,1, \cdots$, we have $R\left(-i t \vartheta^{\prime}\right)=0$ for $t>\gamma_{0}$ from (4.9). Then we deduce from (4.25) and (4.30) that

$$
t \leq C_{1}^{\prime}+C_{2}^{\prime} \log (1+|t|), \quad t>\gamma_{0}
$$

which is impossible. Consequently there exists an integer $j_{0} \geq 0$ such that $R^{j_{0}}\left(\vartheta^{\prime}\right) \neq 0$ and $R^{j}\left(\vartheta^{\prime}\right)=0, j=0, \cdots, j_{0}-1$, if $j_{0} \geq 1$. Suppose that $R^{0}\left(\vartheta^{\prime}\right)=0$. Since $R^{0}\left(\zeta^{\prime}\right) \not \equiv 0$, we take a $\zeta^{0 \prime} \in \boldsymbol{\Xi}^{n-1}-i \dot{\Gamma}$ such that $R^{0}\left(\zeta^{\prime \prime}\right)$ $\neq 0$ and consider the function $f(s, z)$ defined by (4.20). By the same argument as in the case when $S\left(\zeta^{\prime}\right) \equiv 0$, the equation $f(s, z)=0$ has a solution $z(s) \not \equiv 0$ of the form (4.22). Then $t(s)=z(s)^{-1}$ satisfies (4.24). Set $\zeta^{\prime}(s)=s t(s)\left(\zeta^{0 \prime}-\vartheta^{\prime}\right)+t(s) \vartheta^{\prime}$ and choose the path $\kappa: 0<|s|<d$ and $\arg \left(a s^{\nu}\right)=-\pi / 2$. Then we have 
(4.31) $R\left(\zeta^{\prime}(s)\right)=0$ and $\operatorname{Im} \zeta^{\prime}(s)=-|a||s|^{\nu} \vartheta^{\prime}(1+o(1))$ as $s \rightarrow 0$

along the path $\kappa$. In view of (4.25), we have

(4.32) $-h\left(\operatorname{Im} \zeta^{\prime}(s)\right)=|a| \cdot|s|^{\nu} \cdot(1+o(1)) \quad$ as $s \rightarrow 0$ along $\kappa$.

Since $\left|\zeta^{\prime}(s)\right| \leq$ Const $|s|^{\nu}$, we deduce from (4.30) and (4.32) that

$$
|s|^{\nu} \leq C_{1}^{\prime}+C_{2}^{\prime} \log \left(1+|s|^{\nu}\right) \quad \text { as } s \rightarrow 0 \text { along } \kappa \text {. }
$$

Here $C_{1}^{\prime}$ and $C_{2}^{\prime}$ are some new positive constants independent of $s$. Since $\nu$ is a negative rational number, this is impossible. Therefore we conclude that $R^{0}\left(\vartheta^{\prime}\right) \neq 0$.

We shall finally prove the assertion (II). We first note that if we consider the polynomial of the variables $\zeta^{\prime}, \lambda_{1}^{+}, \cdots, \lambda_{p}^{+}$:

$$
\mathscr{R}\left(\zeta^{\prime}, \lambda_{1}^{+}, \cdots, \lambda_{p}^{+}\right)=\operatorname{det}\left(B_{k}\left(\zeta^{\prime}, \lambda_{j}^{+}\right)\right) / \prod_{k<j}\left(\lambda_{k}^{+}-\lambda_{j}^{+}\right),
$$

we have

$$
R\left(\zeta^{\prime}\right)=R\left(\zeta^{\prime}, \lambda_{1}^{+}\left(\zeta^{\prime}\right), \cdots, \lambda_{p}^{+}\left(\zeta^{\prime}\right)\right)
$$

Put

$$
\begin{gathered}
M_{r}=\left\{\left(\xi^{\prime}, \operatorname{Re} s, \operatorname{Im} s, \operatorname{Re} \lambda_{1}, \operatorname{Im} \lambda_{1}, \cdots, \operatorname{Re} \lambda_{m^{\prime}}, \operatorname{Im} \lambda_{m^{\prime}}\right)\right. \\
q_{0}\left(\xi^{\prime}+s \vartheta^{\prime}\right) \sum_{i=1}^{m^{\prime}} \lambda_{i}=-q_{1}\left(\xi^{\prime}+s \vartheta^{\prime}\right), \\
\vdots \\
q^{0}\left(\xi^{\prime}+s \vartheta^{\prime}\right) \prod_{i=1}^{m^{\prime}} \lambda_{i}=(-1)^{m^{\prime}} q_{m^{\prime}}\left(\xi^{\prime}+s \vartheta^{\prime}\right), \\
\operatorname{Im} \lambda_{1}>0, \cdots, \operatorname{Im} \lambda_{p}>0, \operatorname{Im} s<-\gamma_{0},\left|\xi^{\prime}\right|^{2}=r^{2}, \quad \xi^{\prime} \in \mathbb{G}^{n-1}, \\
\left.\mathscr{R}\left(\xi^{\prime}, \lambda_{1}^{+}, \cdots, \lambda_{p}^{+}\right)=0\right\}
\end{gathered}
$$

and

$$
\mu(r)=\sup _{M_{r}}-\operatorname{Im} s,
$$

the supremum being taken over all $-\operatorname{Im} s$ such that $\left(\xi^{\prime}, \operatorname{Re} s, \operatorname{Im} s, \operatorname{Re} \lambda_{1}\right.$, $\left.\operatorname{Im} \lambda_{1}, \cdots, \operatorname{Re} \lambda_{m^{\prime}}, \operatorname{Im} \lambda_{m^{\prime}}\right) \in M_{r}$. Since $R^{0}\left(\vartheta^{\prime}\right) \neq 0$ and $q_{0}\left(\xi^{\prime}+s \vartheta^{\prime}\right) \neq 0$ when $\operatorname{Im} s<-\gamma_{0}$, we have $\mu(r) \not \equiv \infty$. Then it follows from the TarskiSeidenberg theorem that

$$
\mu(r)=a r^{b}(1+o(1)), \quad r \rightarrow+\infty,
$$

where $a$ is real and $b$ is rational. Let us denote by $s\left(\xi^{\prime}\right)$ the zeros of the equation $R\left(\xi^{\prime}+s \vartheta^{\prime}\right)=0$ in $s$. If $\operatorname{Im} s\left(\xi^{\prime}\right)<0$, we have from (4. 30) 
(4. 34) $-\operatorname{Im} s\left(\xi^{\prime}\right)\left(-h\left(-\vartheta^{\prime}\right)\right) \leq C_{1}+C_{2} \log \left(1+\left|\xi^{\prime}+s\left(\xi^{\prime}\right) \vartheta^{\prime}\right|\right)$

because $h\left(\operatorname{Im}\left(\xi^{\prime}+s \vartheta^{\prime}\right)\right)=(-\operatorname{Im} s) h\left(-\vartheta^{\prime}\right)$. When $\operatorname{Im} s\left(\xi^{\prime}\right) \geq 0$, (4. 34) is obvious. Using the Tarski-Seidenberg theorem we have also

$$
\sup _{\left|\xi^{\prime}\right|=r}\left|s\left(\xi^{\prime}\right)\right| \leq \text { Const } r^{d}, \text { as } r \rightarrow+\infty .
$$

Hence we get from (4.33), (4.34) and (4.35)

$$
-h\left(-\vartheta^{\prime}\right) \mu(r) \leq C_{1}+C_{2} \log \left(1+r+C_{3} r^{d}\right) \text { as } r \rightarrow+\infty .
$$

Since $-h\left(-\vartheta^{\prime}\right)>0$, this inequality means that $\mu(r)$ remains bounded when $r \rightarrow+\infty$. This implies that there exists a real $r_{1}$ such that $R\left(\xi^{\prime}+s \vartheta^{\prime}\right) \neq 0$ when $\operatorname{Im} s<\gamma_{1}$ and $\xi^{\prime} \in \boldsymbol{G}^{n-1}$. Thus the proof of Proposition 4.3 is complete.

Assume that the Lopatinski determinant $R\left(\zeta^{\prime}\right)$ of $\left\{P(D) ; B_{j}(D)\right.$, $j=1, \cdots, p$ ) is hyperbolic with respect to $\vartheta^{\prime}$, where $P(D)$ is hyperbolic with respect to $\vartheta=(1,0, \cdots, 0)$. Then the principal part $R^{0}\left(\zeta^{\prime}\right)$ is also hyperbolic with respect to $\vartheta^{\prime}$. Let us denote by $\dot{\Sigma}$ the connected component containing $\vartheta^{\prime}$ of the set $\left\{\xi^{\prime} \in \dot{\Gamma} ; R^{0}\left(\xi^{\prime}\right) \neq 0\right\}$. Then $\dot{\Sigma}$ is an open convex cone in $\Xi^{n-1}$. (See Wakabayashi [12], Lemma 2.3.) Then we have

Lemma 4. 4 ([9]). There exists a constant $\gamma_{1}$ such that

$$
R\left(\zeta^{\prime}\right) \neq 0 \text { for } \zeta^{\prime} \in \mathbb{G}^{n-1}-i \gamma_{1} \vartheta^{\prime}-i \dot{\Sigma} .
$$

Moreover there exists locally bounded functions $C\left(\eta^{\prime}\right)$ and $N\left(\eta^{\prime}\right)$ such that

$$
C\left(t \eta^{\prime}\right)=C\left(\eta^{\prime}\right), N\left(t \eta^{\prime}\right)=N\left(\eta^{\prime}\right) \text { when } t \geq 1, \eta^{\prime} \in-\gamma_{1} \vartheta^{\prime}-\dot{\Sigma}
$$

and that

$$
\begin{gathered}
\left|R\left(\xi^{\prime}+i \eta^{\prime}\right)^{-1}\right| \leq C\left(\eta^{\prime}\right)\left(1+\left|\xi^{\prime}+i \eta^{\prime}\right|\right)^{N\left(\eta^{\prime}\right)}, \\
\xi^{\prime}+i \eta^{\prime} \in \Xi^{n-1}-i \gamma_{1} \vartheta^{\prime}-i \dot{\Sigma} .
\end{gathered}
$$

\section{§. Proof of the Main Theorem}

Let $P(D)$ be a hyperbolic operator with respect to $\vartheta=(1,0, \cdots, 0)$ 
and let $p$ be the number of the roots with positive imaginary part of the equation $P\left(\xi^{\prime}-i s \vartheta^{\prime}, \lambda\right)=0$ in $\lambda$ for $\xi^{\prime} \in \boldsymbol{E}^{n-1}$ and sufficiently large $s$. Let $B_{j}(D), j=1, \cdots, q$, be boundary operators with constant coefficients. Then we have the following two propositions.

Proposition 5.1. Assume that $p=q$ and that the Lopatinski determinant $R\left(\zeta^{\prime}\right)$ of the system $\left\{P(D) ; B_{j}(D), j=1, \cdots, p\right\}$ is hyperbolic with respect to $\vartheta^{\prime}$, if $p \geq 1$. Let $W(x) \in C^{\infty}\left([0, \infty) ; \mathscr{D}^{\prime}\left(\mathbb{R}^{n-1}\right)\right)$ be a solution of the homogeneous problem:

$$
P(D) W(x)=0, \quad x \in \boldsymbol{R}_{+}^{n},\left.\quad B_{j}(D) W(x)\right|_{x_{n}=0}=0, \quad j=1, \cdots, p .
$$

If supp $W \subset\left\{x \in \overline{\boldsymbol{R}_{+}^{n}} ; x_{1}=x \cdot \vartheta \geq 0\right\}$, then $W(x) \equiv 0$.

Proof. Put

$$
\Sigma=\left\{\xi \in \Gamma ; \xi^{\prime} \in \dot{\Sigma}\right\},
$$

where $\Gamma=\Gamma(P, \vartheta)$ (see Section 4). Let $\Gamma^{\prime}$ and $\Sigma^{\prime}$ denote the dual cone of $\Gamma$ and $\Sigma$, respectively. For any positive number $b$, we put

$$
\begin{aligned}
& S_{b}=\left\{x \in \boldsymbol{R}^{n} ; b \leq|x| \leq b+1, x_{n} \geq 0, x_{1} \geq 0\right\}, \\
& \left.\dot{S}_{b}=\left\{x^{\prime} \in \boldsymbol{R}^{n-1} ;\left(x^{\prime}, 0\right) \in S_{b} \text { (i.e. } b \leq\left|x^{\prime}\right| \leq b+1\right), x_{1} \geq 0\right\}, \\
& \Gamma_{S_{b}}^{\prime}=\Gamma^{\prime}+S_{b}, \quad \dot{\Gamma}_{S_{b}}^{\prime}=\left\{x^{\prime} \in \boldsymbol{R}^{n-1} ;\left(x^{\prime}, 0\right) \in \Gamma_{S_{b}}^{\prime}\right\}
\end{aligned}
$$

and

$$
D_{b}=\left\{\left[\Sigma^{\prime}+\left(\dot{\Gamma}_{S_{b}}^{\prime} \cup \dot{S}_{b}\right)\right] \cup \Gamma_{S_{b}}^{\prime}\right\} \cap \overline{\boldsymbol{R}_{+}^{n}} .
$$

Since both $\Gamma$ and $\Sigma$ are closed proper cone with its vertex at the origin such that $x \cdot \vartheta>0$ on $\Gamma-\{0\}$, there exists a number $b>0$ for an arbitrarily given $a>0$ such that $D_{b}$ and $B_{a}=\left\{x \in \mathbb{R}^{n}\right.$; $\left.|x| \leq a, x_{1} \geq 0, x_{n} \geq 0\right\}$ are disjoint.

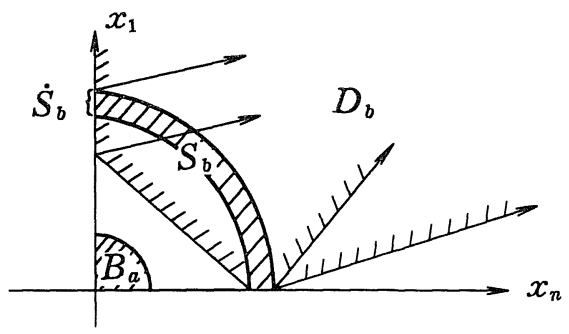

Choose such a number $b>0$. Let $\phi \in C_{0}^{\infty}\left(\left\{x \in \boldsymbol{R}^{n} ;|x| \leq b+1\right\}\right)$ be equal to 1 in $\left\{x \in \mathbb{R}^{n} ;|x| \leq b\right\}$. Then we have

$$
P(D)(\phi W)=f(x), x_{n}>0,
$$




$$
\left.B_{j}(D)(\phi W)\right|_{x_{n}=0}=g_{j}\left(x^{\prime}\right), \quad j=1, \cdots, p,
$$

where $\phi W$ and $f$ are $C^{\infty}$ function of $x_{n}$ in $[0, \infty)$ with values in $\mathcal{E}^{\prime}\left(\boldsymbol{R}^{n-1}\right), g_{j}\left(x^{\prime}\right)$ belongs to $\mathcal{E}^{\prime}\left(R^{n-1}\right)$ and

$$
\begin{aligned}
& \operatorname{supp} f(x) \subset S_{b}, \quad x_{n} \geq 0, \\
& \operatorname{supp} g_{j}\left(x^{\prime}\right) \subset \dot{S}_{b} .
\end{aligned}
$$

Taking partial Fourier-Laplace transforms of both sides of (5.1) and (5.2), we obtain a system of ordinary differential equations with a parameter $\zeta^{\prime}$ :

$$
\begin{aligned}
& P\left(\zeta^{\prime}, D_{n}\right) \widehat{\phi W}\left(\zeta^{\prime}, x_{n}\right)=\widehat{f}\left(\zeta^{\prime}, x_{n}\right), \quad x_{n}>0, \\
& \left.B_{j}\left(\zeta^{\prime}, D_{n}\right) W\left(\zeta^{\prime}, x_{n}\right)\right|_{x_{n}=0}=\widehat{g}_{j}\left(\zeta^{\prime}\right), \quad j=1, \cdots, p .
\end{aligned}
$$

$R\left(\zeta^{\prime}\right)$ is, by assumption, hyperbolic and so $R\left(\zeta^{\prime}\right) \neq 0$ for $\zeta^{\prime} \in \mathbb{E}^{n-1}-i \gamma_{1} \vartheta^{\prime}$ $-i \dot{\Sigma}$. Since $\widehat{\phi W}\left(\zeta^{\prime}, \cdot\right), \widehat{f}\left(\zeta^{\prime}, \cdot\right) \in C_{(o)}^{\infty}\left(\overline{\boldsymbol{R}_{+}^{1}}\right)$ for every $\zeta^{\prime}$, we can write, applying Lemma 3. 3,

$$
\widehat{\phi W}\left(\zeta^{\prime}, x_{n}\right)=v_{1}\left(\zeta^{\prime}, x_{n}\right)+v_{2}\left(\zeta^{\prime}, x_{n}\right), x_{n} \geq 0
$$

where

$$
\begin{gathered}
v_{1}\left(\zeta^{\prime}, x_{n}\right)=(2 \pi)^{-1} \int \exp \left(i x_{n} \xi_{n}\right) \mathscr{F}_{x_{n}}\left[\widehat{f}_{0}\right]\left(\zeta^{\prime}, \xi_{n}\right)\left(P\left(\zeta^{\prime}, \xi_{n}\right)\right)^{-1} d \xi_{n} \\
v_{2}\left(\zeta^{\prime}, x_{n}\right)=(2 \pi i)^{-1} \sum_{j, k=1}^{p} \int_{r\left(\zeta^{\prime}\right)} \exp \left(i x_{n} \lambda\right) \lambda^{k-1}\left(P_{+}\left(\zeta^{\prime}, \lambda\right)\right)^{-1} d \lambda \\
\times R_{j k}\left(\zeta^{\prime}\right)\left[g_{j}\left(\zeta^{\prime}\right)-\left.B_{j}\left(\zeta^{\prime}, D_{n}\right) v_{1}\left(\zeta^{\prime}, x_{n}\right)\right|_{x_{n}=0}\right]\left(R\left(\zeta^{\prime}\right)\right)^{-1}
\end{gathered}
$$

Here $R_{j k}\left(\zeta^{\prime}\right)$ is the $(j, k)$ cofactor of the matrix

$$
\left((2 \pi i)^{-1} \int_{r\left(\zeta^{\prime}\right)} B_{j}\left(\zeta^{\prime}, \lambda\right) \lambda^{k-1}\left(P_{+}\left(\zeta^{\prime}, \lambda\right)\right)^{-1} d \lambda\right)_{j, k=1, \ldots, p},
$$

$\widehat{f}_{0}\left(\zeta^{\prime}, x_{n}\right)$ is a compactly supported $C^{\infty}$ extension of $\hat{f}\left(\zeta^{\prime}, x_{n}\right)$ to $x_{n}<0$ and

$$
\mathscr{I}_{x_{n}}\left[\widehat{f}_{0}\right]\left(\zeta^{\prime}, \xi_{n}\right)=\int_{-\infty}^{+\infty} \exp \left(-i x_{n} \xi_{n}\right) \widehat{f}_{0}\left(\zeta^{\prime}, x_{n}\right) d x_{n}
$$

If $p=0$, that is, if the equation $P\left(\zeta^{\prime}, \lambda\right)=0$ in $\lambda$ has only roots $\lambda\left(\zeta^{\prime}\right)$ with $\operatorname{Im} \lambda\left(\zeta^{\prime}\right)<0$ for every $\zeta^{\prime} \in \boldsymbol{\Xi}^{n-1}-i \gamma_{1} \vartheta^{\prime}-i \dot{\Sigma}$, we have

$$
\widehat{\phi W}\left(\zeta^{\prime}, x_{n}\right)=v_{1}\left(\zeta^{\prime}, x_{n}\right) \text { for } x_{n} \geq 0 \text {. }
$$


Therefore we define $v_{2}\left(\zeta^{\prime}, x_{n}\right) \equiv 0$ in this case. Now we have from (5. 7)

$$
(\phi W)(x)=\mathscr{F}_{\zeta^{\prime}}^{-1}\left[v_{1}\left(\zeta^{\prime}, x_{n}\right)\right]+\mathscr{F}_{\zeta^{\prime}}^{-1}\left[v_{2}\left(\zeta^{\prime}, x_{n}\right)\right]
$$

where $\mathscr{F}_{\zeta^{\prime}}^{-1}$ denotes the inverse Fourier-Laplace transform in $\zeta^{\prime}$. From (5. 8) and (5.10) we find

$$
\text { (5. 12) } \mathscr{F} \zeta^{\prime}\left[v_{1}\left(\zeta^{\prime}, x_{n}\right)\right]=\mathscr{F} \zeta^{\prime}\left[(P(\zeta))^{-1} \widehat{f}_{0}(\zeta)\right]=\mathscr{F}^{-1} \zeta^{\prime}\left[(P(\zeta))^{-1}\right] * f_{0} .
$$

Since $\mathscr{F}_{\zeta^{\prime}}^{-1}\left[(P(\zeta))^{-1}\right]$ is the unique fundamental solution of the Cauchy problem for $P(D)$ such that the support is contained in $\Gamma^{\prime}$, we obtain using (5.3)

$$
\operatorname{supp} \mathscr{F}_{\zeta^{\prime}}^{-1}\left[v_{1}\left(\zeta^{\prime}, x_{n}\right)\right] \cap \overline{\boldsymbol{R}_{+}^{n}} \subset \Gamma^{\prime}+S_{b} .
$$

In particular (5.13) implies that

$$
\begin{aligned}
\operatorname{supp} & \left(\left.B_{j}(D) \mathscr{I}_{\zeta^{\prime}}^{-1}\left[v_{1}\left(\zeta^{\prime}, x_{n}\right)\right]\right|_{x_{n}=0}\right) \\
& \subset\left\{x^{\prime} \in \boldsymbol{R}^{n-1} ;\left(x^{\prime}, 0\right) \in \Gamma^{\prime}+S_{b}\right\}=\dot{\Gamma}_{S_{b}}^{\prime} .
\end{aligned}
$$

In view of Lemma 4.4, we have applying the Paley-Wiener-Schwartz theorem ${ }^{7}$

$$
\begin{gathered}
\operatorname{supp} \mathscr{F}^{-1} \zeta^{\prime}\left[\int_{\tau\left(\zeta^{\prime}\right)} \exp \left(i x_{n} \lambda\right) \lambda^{k-1}\left(P_{+}\left(\zeta^{\prime}, \lambda\right)\right)^{-1} d \lambda\right. \\
\left.\times R_{j k}\left(\zeta^{\prime}\right)\left(R\left(\zeta^{\prime}\right)\right)^{-1}\right] \subset \Sigma^{\prime} .
\end{gathered}
$$

From (5.4), (5.9), (5.14) and (5.15), we find

$$
\begin{aligned}
& \operatorname{supp} \mathscr{F}_{\zeta^{\prime}}^{-1}\left[v_{2}\left(\zeta^{\prime}, x_{n}\right)\right] \cap \overline{\boldsymbol{R}_{+}^{n}} \\
& \subset\left\{\Sigma^{\prime}+\bigcup_{j=1}^{p} \operatorname{supp}\left[\left.\left(g_{j}-B_{j}(D) v_{1}\right)\right|_{x_{n}=0} \otimes \delta\left(x_{n}\right)\right] \cap \overline{\boldsymbol{R}_{+}^{n}}\right. \\
& \subset\left\{\Sigma^{\prime}+\left(S_{b} \cup \Gamma_{S_{b}}^{\prime}\right)\right\} \cap \overline{\boldsymbol{R}_{+}^{n}} .
\end{aligned}
$$

From (5.11), (5.13) and (5.16) we conclude that

$$
\operatorname{supp} \phi W \subset D_{b} \text {. }
$$

Since $\phi(x)=1$ on $B_{a}$ and $B_{a} \cap D_{b}=\varnothing,(5.17)$ means that $W(x) \equiv 0$ in $B_{a}$. Letting $a \rightarrow+\infty$, we get $W(x) \equiv 0$ in $\boldsymbol{R}_{+}^{n}$. This completes the proof.

7) For example, see Theorem 2.6 in [1] or Lemma 2.3 in [9]. 
Under the situation at the beginning of this section we have

Proposition 5.2. If $p>q$, there exists a non trivial solution $W(x) \in C^{\infty}\left([0, \infty) ; \mathscr{D}^{\prime}\left(\boldsymbol{R}^{n-1}\right)\right)$ of the following homogeneous problem whose support is contained in some closed proper cone $K_{1}$ with its vertex at the origin such that $x \cdot \vartheta>0$ on $K_{1}-\{0\}$.

$$
\begin{gathered}
P(D) W=0, \quad x_{n}>0, \\
\left.B_{j}(D) W\right|_{x_{n}=0}=0, \quad j=1, \cdots, q .
\end{gathered}
$$

Proof. Consider the matrix $A\left(\zeta^{\prime}\right)=\left(a_{j k}\left(\zeta^{\prime}\right)\right)_{\substack{j=1, \ldots, q \\ k=1, \ldots, p}}$ where

$$
a_{j k}\left(\zeta^{\prime}\right)=(2 \pi i)^{-1} \int_{r\left(\zeta^{\prime}\right)} B_{j}\left(\zeta^{\prime}, \lambda\right) \lambda^{k-1}\left(P_{+}\left(\zeta^{\prime}, \lambda\right)\right)^{-1} d \lambda .
$$

Assume that rank $A\left(\zeta^{\prime}\right)=r(0 \leq r \leq q)$, that is, some $r$-rowed minor $\Delta\left(\zeta^{\prime}\right)$ of $A\left(\zeta^{\prime}\right)$ is not identically zero in $\boldsymbol{\Xi}^{n-1}-i \gamma_{0} \vartheta^{\prime}-i \dot{\Gamma}$ when $1 \leq r \leq q$ and every $(r+1), \cdots, q$-rowed minor of $A\left(\zeta^{\prime}\right)$ is identically zero in $\boldsymbol{\xi}^{n-1}-i \gamma_{0} \vartheta^{\prime}-i \dot{\Gamma}$ when $1 \leq r \leq q-1$. When $r \neq 0$, i.e., $A\left(\zeta^{\prime}\right) \not \equiv 0$, we may assume without loss of generality that

$$
\Delta\left(\zeta^{\prime}\right)=\operatorname{det}\left(a_{j k}\left(\zeta^{\prime}\right)\right)_{j, k=1, \cdots, r} .
$$

When $1 \leq r \leq q$, we put

$$
\begin{aligned}
& \widehat{W}\left(\zeta^{\prime}, x_{n}\right)=-\sum_{j, k=1}^{r} \Delta_{j k}\left(\zeta^{\prime}\right) a_{j r+1}\left(\zeta^{\prime}\right) \\
& \quad \times\left\{(2 \pi i)^{-1} \int_{r\left(\zeta^{\prime}\right)} \exp \left(i x_{n} \lambda\right) \lambda^{k-1}\left(P_{+}\left(\zeta^{\prime}, \lambda\right)\right)^{-1} d \lambda\right\} \\
& \quad+\Delta\left(\zeta^{\prime}\right)(2 \pi i)^{-1} \int_{r\left(\zeta^{\prime}\right)} \exp \left(i x_{n} \lambda\right) \lambda^{r}\left(P_{+}\left(\zeta^{\prime}, \lambda\right)\right)^{-1} d \lambda,
\end{aligned}
$$

where $\Delta_{j k}\left(\zeta^{\prime}\right)$ is the $(j, k)$ cofactor of $\left(a_{j k}\left(\zeta^{\prime}\right)\right)_{j, k=1, \ldots, r}$. When $r=0$, put

$$
\widehat{W}\left(\zeta^{\prime}, x_{n}\right)=(2 \pi i)^{-1} \int_{r\left(\zeta^{\prime}\right)} \exp \left(i x_{n} \lambda\right)\left(P_{+}\left(\zeta^{\prime}, \lambda\right)\right)^{-1} d \lambda
$$

Since

$$
(2 \pi i)^{-1} \int_{r_{\left(\zeta^{\prime}\right)}} \exp \left(i x_{n} \lambda\right) \lambda^{j-1}\left(P_{+}\left(\zeta^{\prime}, \lambda\right)\right)^{-1} d \lambda, \quad j=1, \cdots, p
$$

are linearly independent functions of $x_{n}$ and $\Delta\left(\zeta^{\prime}\right) \not \equiv$ in $\boldsymbol{\Xi}^{n-1}-i \gamma_{0} \vartheta^{\prime}-i \dot{\Gamma}$, 
we have $\widehat{W}\left(\zeta^{\prime}, x_{n}\right) \not \equiv 0$. Since $\widehat{W}\left(\zeta^{\prime}, x_{n}\right)$ is analytic in $\zeta^{\prime}$, we have $\widehat{W}\left(\zeta^{\prime}, x_{n}\right) \neq 0$ in $\boldsymbol{R}_{+}^{n}$. It is easy to see that $W(x)$ belongs to $C^{\infty}([0, \infty)$; $\mathscr{D}^{\prime}\left(\boldsymbol{R}^{n-1}\right)$ ) and satisfies (5.18) and (5.19). Moreover, using the PaleyWiener-Schwartz theorem, we see that the support of $W(x)$ is contained in $K_{1} \cap \overline{\boldsymbol{R}_{+}^{n}}$ where $K_{1}$ is some closed proper cone such that $x \cdot \vartheta>0$ on $K_{1}-\{0\}$. This completes the proof.

Let us now complete our proof of the Main Theorem which is stated in Introduction.

Proof of the Main Theorem. In Proposition 2.1 we proved that the existence of certain fundamental solution $E(x, y)$ of the mixed problem (1.3)-(1.5) for some $y=\left(0, y^{\prime \prime}\right) \in \boldsymbol{R}_{+}^{n}$ (i.e., $E$ satisfies (1.8) and (1.9) and its support is contained in $(K+y) \cap \overline{\boldsymbol{R}_{+}^{n}}$ where $K$ is some closed proper cone such that $x \cdot \vartheta>0$ on $K-\{0\})$ implies the hyperbolicity of the characteristic polynomial $P(\xi)$ with respect to $\vartheta=(1,0, \cdots, 0)$. The hyperbolicity of $P(\xi)$ determines the number $p$. Next the uniqueness of the system $\left\{F_{j}(x), j=1, \cdots, q\right\}$ implies that the number $q$ of boundary operators $B_{j}(D)$ must be greater than or equal to $p$, because if $q<p$ the system $\left\{F_{j}(x), j=1, \cdots, q\right\}$ is not unique by Proposition 5. 2. Now suppose that $q>p$. When $p=0$, Proposition 5. 1 shows that if $P(D) F_{1}(x)=0$ and $\operatorname{supp} F_{1} \subset\left\{x \in \overline{\boldsymbol{R}_{+}^{n}}, x_{1} \geq 0\right\}$ then $F_{1}(x) \equiv 0$. This contradicts the definition of $F_{1}(x)$. In fact $F_{1}(x)$ must satisfies the equation $\left.B_{1}(D) F_{1}(x)\right|_{x_{n}=0}=\delta\left(x^{\prime}\right)$. When $p \geq 1$, the existence of $F_{j}(x), j=1, \cdots, p$, implies that the Lopatinski determinant of the system $\left\{P(D) ; B_{j}(D), j=1, \cdots, p\right\}$ is hyperbolic with respect to $\vartheta^{\prime}$. Since $F_{p+1}(x)$ satisfies the equations:

$$
P(D) F_{p+1}(x)=0 \text { in } \boldsymbol{R}_{+}^{n},\left.\quad B_{j}(D) F_{p+1}(x)\right|_{x_{n}=0}=0, \quad j=1, \cdots, p,
$$

and supp $F_{p+1} \subset K \cap \overline{\boldsymbol{R}_{+}^{n}}$, it follows from Proposition 5.1 that $F_{p+1}(x) \equiv 0$ in $\overline{\boldsymbol{R}_{+}^{n}}$. This gives a contradiction because $F_{p+1}(x)$ satisfies by definition the equation: $\left.B_{p+1}(D) F_{p+1}(x)\right|_{x_{n}+0}=\delta\left(x^{\prime}\right)$. Thus we conclude that the number $q$ of boundary operators $B_{j}(D)$ must be equal to $p$. If $p=0$, this means that such boundary conditions cannot be imposed. If $p \geq 1$, we see by Proposition 4.3 that the Lopatinski determinant $R\left(\zeta^{\prime}\right)$ of the 
system $\left\{P(D) ; B_{j}(D), j=1, \cdots, p\right\}$ is hyperbolic with respect to $\vartheta^{\prime}$. The proof is complete.

\section{§ 6. Proof of Sufficiency}

First we construct a system $\left\{E(x, y) ; F_{j}(x), j=1, \cdots, q\right\}$ of fundamental solutions of the mixed problem (1.3)-(1.5) for any given system $\left\{P(D) ; B_{j}(D), j=1, \cdots, q\right\}$ of differential operators satisfying the conditions:

(I) $P(\xi)$ is a hyperbolic polynomial with respect to $\vartheta=(1,0, \cdots, 0)$, that is, $P(\xi)$ satisfies (1.1) and (1.2),

(II) the number $q$ of the boundary operators $B_{j}(D)$ is equal to the number $p$ of the roots with positive imaginary part of the equation $P\left(\xi^{\prime}-i s \vartheta^{\prime}, \lambda\right)=0$ in $\lambda$ when $\xi^{\prime} \in \boldsymbol{\Xi}^{n-1}$ and $s>\gamma_{0}$,

(III) the Lopatinski determinant $R\left(\zeta^{\prime}\right)$ of $\left\{P(D) ; B_{j}(D), j=1, \cdots, p\right\}$ is hyperbolic with respect to $\vartheta^{\prime}$, that is, $R\left(\zeta^{\prime}\right)$ satisfies (1.6) and (1.7).

Let $G(x)$ be the unique fundamental solution of the Cauchy problem for $P(D)$ in the half space $x \cdot \vartheta>0 . G(x-y)$ is given in the form

$$
\begin{gathered}
\langle G(x-y), \check{\phi}(x)\rangle=(2 \pi)^{-n} \int_{z^{n}}(P(\xi+i \eta))^{-1} \widehat{\phi}(\xi+i \eta) d \xi, \\
\eta \in-\gamma_{0} \vartheta-\Gamma,
\end{gathered}
$$

where $\check{\phi}(x)=\phi(-x) \in \mathscr{D}\left(\boldsymbol{R}^{n}\right)$ and $\hat{\phi}(\xi+i \eta)$ is the Fourier-Laplace transform of $\phi$. We may assume that $\phi$ is of the form: $\phi(x)=\psi\left(x^{\prime}\right) \rho\left(x_{n}\right)$, $\psi \in \mathscr{D}\left(\boldsymbol{R}^{n-1}\right)$ and $\rho \in \mathscr{D}\left(R^{1}\right)$, since the set of all test functions of this form is dense in $\mathscr{D}\left(\boldsymbol{R}^{n}\right)$. Now we wish to show that $G(x-y)$ $\in C^{\infty}\left(\left[0, y_{n}\right) ; \mathscr{D}^{\prime}\left(R_{x^{\prime}}^{n-1}\right)\right) \cap C^{\infty}\left(\left(y_{n}, \infty\right) ; \mathscr{D}^{\prime}\left(R_{x^{\prime}}^{n-1}\right)\right){ }^{8)} \quad$ To do so we write in Section 4

$$
P(\xi+i \eta)=q_{0}\left(\xi^{\prime}+i \eta^{\prime}\right)\left(\xi_{n}+i \eta_{n}\right)^{m^{\prime}}+\sum_{l=1}^{m^{\prime}} q_{l}\left(\xi^{\prime}+i \eta^{\prime}\right)\left(\xi_{n}+i \eta_{n}\right)^{m^{\prime}-l} .
$$

If $m^{\prime}=0, P(\xi+i \eta)=P\left(\xi^{\prime}+i \eta^{\prime}\right)$. In this case we do not consider any boundary condition and so $E(x, y)=G(x-y)$. Then we have

8) Let $I$ be an interval in $\boldsymbol{R}_{+}^{1}$ and let $u \in \mathscr{D}^{\prime}\left(\boldsymbol{R}_{+}^{n}\right)$. For simplicity we write $u \in C^{\infty}(I$; $\left.\mathscr{D}^{\prime}\left(\boldsymbol{R}_{x^{\prime}}^{n-1}\right)\right)$ in place of $\left.u\right|_{\boldsymbol{R}_{x^{\prime}}^{n-1} \times I^{\circ}} \in C^{\infty}\left(I ; \mathscr{D}^{\prime}\left(R_{x^{\prime}}^{n-1}\right)\right)$ where $\left.u\right|_{\boldsymbol{R}_{x^{\prime}}^{n-1} \times I^{\circ}}$ is the restriction of $u$ to $\boldsymbol{R}_{x^{\prime}}^{n-1} \times I$. 
(6. 3) $\left\langle E(x, y), \breve{\psi}\left(x^{\prime}\right) \rho\left(x_{n}\right)\right\rangle$

$$
\begin{aligned}
& =(2 \pi)^{-n+1}\left\{\int_{\nabla^{n}} \exp \left\{-i y^{\prime}\left(\xi^{\prime}+i \eta^{\prime}\right)\right\}\left(P\left(\xi^{\prime}+i \eta^{\prime}\right)\right)^{-1}\right. \\
& \left.\times \widehat{\psi}\left(\xi^{\prime}+i \eta^{\prime}\right) d \xi^{\prime}\right\}\left\langle\delta\left(x_{n}-y_{n}\right), \rho\left(x_{n}\right)\right\rangle .
\end{aligned}
$$

Thus, in this case it is obvious that $E(\cdot, y) \in C^{\infty}\left(\left[0, y_{n}\right) ; \mathscr{D}^{\prime}\left(\boldsymbol{R}^{n-1}\right)\right)$ $\cap C^{\infty}\left(\left(y_{n}, \infty\right) ; \mathscr{D}^{\prime}\left(\boldsymbol{R}^{n-1}\right)\right)$.

Let us consider the case where $m^{\prime} \geq 1$. Take $\chi \in C_{0}^{\infty}\left(\left\{\xi_{n} \in \boldsymbol{g}^{1}\right.\right.$; $\left.\left.\left|\xi_{n}\right|<2\right\}\right)$ such that $\chi\left(\xi_{n}\right)=1$ when $\left|\xi_{n}\right|<1$. Using Lebesgue's dominated convergence theorem and Fubini's theorem we find

$$
\begin{aligned}
\left\langle G(x-y), \breve{\psi}\left(x^{\prime}\right) \check{\rho}\left(x_{n}\right)\right\rangle \\
=\lim _{\varepsilon \downarrow 0}(2 \pi)^{-n} \int \exp \{-i y(\xi+i \eta)\} \chi\left(\varepsilon \xi_{n}\right)(P(\xi+i \eta))^{-1} \\
\quad \times \widehat{\psi}\left(\xi^{\prime}+i \eta^{\prime}\right) \hat{\rho}\left(\xi_{n}+i \eta_{n}\right) d \xi \\
=\lim _{\varepsilon \downarrow 0}(2 \pi)^{-n} \int_{-\infty}^{+\infty}\left[\int_{\Sigma^{n-1}} \exp \left\{-i y^{\prime}\left(\xi^{\prime}+i \eta^{\prime}\right)\right\}\right. \\
\left.\quad \times \widehat{\psi}\left(\xi^{\prime}+i \eta^{\prime}\right) I_{\varepsilon}\left(x_{n}, \xi^{\prime}+i \eta^{\prime}\right) d \xi^{\prime}\right] \check{\rho}\left(x_{n}\right) d x_{n},
\end{aligned}
$$

where

$$
\begin{aligned}
I_{\varepsilon}\left(x_{n}, \xi^{\prime}+i \eta^{\prime}\right)= & \int_{-\infty}^{+\infty} \exp \left\{i\left(x_{n}-y_{n}\right)\left(\xi_{n}+i \eta_{n}\right)\right\} \chi\left(\varepsilon \xi_{n}\right) \\
& \times(P(\xi+i \eta))^{-1} d \xi_{n}
\end{aligned}
$$

In order to derive an expression which is valid when $\varepsilon \rightarrow 0$, we shall first establish the following estimate.

$$
\begin{gathered}
\left|\left(\partial / \partial \xi_{n}\right)^{\alpha}(P(\xi+i \eta))^{-1}\right| \leq M_{\alpha} \frac{\left(1+\left|\xi^{\prime}+i \eta^{\prime}\right|\right)^{k_{\alpha}}}{\left(1+\left|\xi_{n}+i \eta_{n}\right|\right)^{m^{\prime}+\alpha}}, \\
\alpha=0,1,2, \cdots,
\end{gathered}
$$

where $M_{\alpha}$ and $k_{\alpha}$ are some positive constants independent of $\xi+i \eta \in \boldsymbol{G}^{n}$ $-i \gamma_{0} \vartheta-i \Gamma$.

In fact, we have for some positive constants $C_{1}$ and $C_{2} \geq 1$

$$
\begin{aligned}
& \left|q_{0}\left(\xi^{\prime}+i \eta^{\prime}\right)\right| \geq C_{1} \quad(\text { see }(4.4)) \\
& \text { and }\left|q_{l}\left(\xi^{\prime}+i \eta^{\prime}\right)\right|<C_{2}\left(1+\left|\xi^{\prime}+i \eta^{\prime}\right|\right)^{m-m^{\prime}-l} .
\end{aligned}
$$


Then it follows from (6.2) that

$$
|P(\xi+i \eta)| \geq C_{1}\left|\xi_{n}+i \eta_{n}\right|^{m^{\prime}}\left\{1-\sum_{l=1}^{m^{\prime}}\left|\frac{q_{l}\left(\xi^{\prime}+i \eta^{\prime}\right)}{q_{0}\left(\xi^{\prime}+i \eta^{\prime}\right)}\right|\left|\xi_{n}+i \eta_{n}\right|^{-l}\right\} .
$$

Hence if $\left|\xi_{n}+i \eta_{n}\right|>C_{3}\left(1+\left|\xi^{\prime}+i \eta^{\prime}\right|\right)^{m-m^{\prime}-1}$ with $C_{3}=2 m^{\prime} C_{1}^{-1} C_{2}$, we have from (6.7)

$$
\sum_{l=1}^{m^{\prime}}\left|q_{l}\left(\xi^{\prime}+i \eta^{\prime}\right) / q_{0}\left(\xi^{\prime}+i \eta^{\prime}\right)\right|\left|\xi_{n}+i \eta_{n}\right|^{-l} \leq 1 / 2
$$

and so

$$
|P(\xi+i \eta)|^{-1} \leq C_{4}\left(1+\left|\xi_{n}+i \eta_{n}\right|\right)^{-m^{\prime}} .
$$

On the other hand, if $\left|\xi_{n}+i \eta_{n}\right|<C_{3}\left(1+\left|\xi^{\prime}+i \eta^{\prime}\right|\right)^{-m^{\prime}}$, we have

(6. 9) $|P(\xi+i \eta)|^{-1} \leq C_{5} \leq C_{6}\left(1+\left|\xi^{\prime}+i \eta^{\prime}\right|\right)^{m^{\prime}\left(m-m^{\prime}+1\right)}\left(1+\left|\xi_{n}+i \eta_{n}\right|\right)^{-m^{\prime}}$.

From (6.8) and (6.9) we find (6.6) for $\alpha=0$ with $M_{0}=\operatorname{Max}\left(C_{5}\left(2 C_{3}\right)^{m^{\prime}}\right.$, $\left.C_{4}\right)$ and $k_{0}=m^{\prime}\left(m-m^{\prime}+1\right)$. We show (6.6) for $\alpha \geq 1$ by induction. Now we have

$$
\begin{aligned}
& \left|\left(\partial / \partial \xi_{n}\right) P(\xi+i \eta)\right|=\mid m^{\prime} q_{0}\left(\xi^{\prime}+i \eta^{\prime}\right)\left(\xi_{n}+i \eta_{n}\right)^{m^{\prime}-1} \\
& \quad+\sum_{l=1}^{m^{\prime}}\left(m^{\prime}-l-1\right) q_{l}\left(\xi^{\prime}+i \eta^{\prime}\right)\left(\xi_{n}+i \eta_{n}\right)^{m^{\prime}-l-1} \mid \\
& \leq C_{7}\left(1+\left|\xi^{\prime}+i \eta^{\prime}\right|\right)^{m}\left(1+\left|\xi_{n}+i \eta_{n}\right|\right)^{m^{\prime}-1} .
\end{aligned}
$$

Hence

$$
\begin{gathered}
\left|\left(\partial / \partial \xi_{n}\right)(P(\xi+i \eta))^{-1}\right|=\left|\left(\partial / \partial \xi_{n}\right) P(\xi+i \eta)\right||P(\xi+i \eta)|^{-2} \\
\leq M_{1}\left(1+\left|\xi^{\prime}+i \eta^{\prime}\right|\right)^{k_{1}}\left(1+\left|\xi_{n}+i \eta_{n}\right|\right)^{-m^{\prime}-1}
\end{gathered}
$$

Suppose that (6.6) holds for $0 \leq l \leq \alpha-1$. From the relation

$$
0=\left(\partial / \partial \xi_{n}\right)^{\alpha}\left(P^{-1} \cdot P\right)=\sum_{l=0}^{\alpha}\left(\begin{array}{l}
\alpha \\
l
\end{array}\right)\left\{\left(\partial / \partial \xi_{n}\right)^{l}\left(P^{-1}\right)\right\}\left\{\left(\partial / \partial \xi_{n}\right)^{\alpha-l} P\right\}
$$

we get

$$
\left(\partial / \partial \xi_{n}\right)^{\alpha}\left(P^{-1}\right)=-\sum_{l=0}^{a-1}\left(\begin{array}{l}
\alpha \\
l
\end{array}\right)\left\{\left(\partial / \partial \xi_{n}\right)^{l}\left(P^{-1}\right)\right\}\left\{\left(\partial / \partial \xi_{n}\right)^{\alpha-l} P\right\} P^{-1} .
$$

Now we have

$$
\left|\left(\partial / \partial \xi_{n}\right)^{\alpha-l} P(\xi+i \eta)\right| \leq C\left(1+\left|\xi^{\prime}+i \eta^{\prime}\right|\right)^{m}\left(1+\left|\xi_{n}+i \eta_{n}\right|\right)^{m^{\prime}-\alpha+l}
$$

and by the induction assumption 


$$
\left|\left(\partial / \partial \xi_{n}\right)^{l}(P(\xi+i \eta))^{-1}\right| \leq M\left(1+\left|\xi^{\prime}+i \eta^{\prime}\right|\right)^{k_{l}}\left(1+\left|\xi_{n}+i \eta_{n}\right|\right)^{-m^{\prime}-l}
$$

$l=0,1, \cdots, \alpha-1$. Applying these estimates to the right-hand side of (6.10), we have (6.6) for $\alpha$.

We shall now return to the integrals (6.4) and (6.5). Using repeatedly the relation

$$
\begin{aligned}
& \exp \left\{i\left(x_{n}-y_{n}\right)\left(\xi_{n}+i \eta_{n}\right)\right\} \\
& \quad=-i\left(x_{n}-y_{n}\right)^{-1}\left(\partial / \partial \xi_{n}\right) \exp \left\{i\left(x_{n}-y_{n}\right)\left(\xi_{n}+i \eta_{n}\right)\right\}
\end{aligned}
$$

and integrating by parts, we obtain

$$
\text { (6.11) } \begin{aligned}
I_{\varepsilon} & \left(x_{n}, \xi^{\prime}+i \eta^{\prime}\right)=\left(y_{n}-x_{n}\right)^{-\alpha}\left[\int_{-\infty}^{+\infty} \exp \left\{i\left(x_{n}-y_{n}\right)\left(\xi_{n}+i \eta\right)\right\} \chi\left(\varepsilon \xi_{n}\right)\right. \\
& \left.\times\left(-i \partial / \partial \xi_{n}\right)^{\alpha}(P(\xi+i \eta))^{-1} d \xi_{n}+J_{\varepsilon}\left(x_{n}, \xi^{\prime}+i \eta^{\prime}\right)\right], \quad x_{n} \neq y_{n},
\end{aligned}
$$

where

$$
\text { (6.12) } \begin{aligned}
J_{\varepsilon} & \left(x_{n}, \xi^{\prime}+i \eta^{\prime}\right)=\sum_{l=1}^{1 a} \varepsilon^{l} \int_{-\infty}^{+\infty} \exp \left\{i\left(x_{n}-y_{n}\right)\left(\xi_{n}+i \eta_{n}\right)\right\} \chi^{(l)}\left(\varepsilon \xi_{n}\right) \\
& \times\left[\left(-i \partial / \partial \xi_{n}\right)^{a-l}(P(\xi+i \eta))^{-1}\right] d \xi_{n}, \quad\left(\chi^{(l)}=\left(-i \partial / \partial \xi_{n}\right)^{l} \chi\right) .
\end{aligned}
$$

The estimate (6.6) gives

$$
\begin{aligned}
& \text { (6.13) }\left|\exp \left\{i\left(x_{n}-y_{n}\right)\left(\xi_{n}+i \eta_{n}\right)\right\} \chi^{(l)}\left(\varepsilon \xi_{n}\right)\left[\left(-i \partial / \partial \xi_{n}\right)^{\alpha-l}(P(\xi+i \eta))^{-1}\right]\right| \\
& \leq M_{\alpha-l} \exp \left\{\left(y_{n}-x_{n}\right) \eta_{n}\right\}\left(1+\left|\xi^{\prime}+i \eta^{\prime}\right|\right)^{k_{a}-l} \\
& \quad \times\left(1+\left|\xi_{n}+i \eta_{n}\right|\right)^{-m^{\prime}-a+l} .
\end{aligned}
$$

Using Schwarz inequality and (6.13) we find

$$
\begin{aligned}
\left|J_{\varepsilon}\left(x_{n}, \xi^{\prime}+i \eta^{\prime}\right)\right| \leq C \varepsilon\left(1+\left|\xi^{\prime}+i \eta^{\prime}\right|\right)^{k} \\
\times\left\{\int_{-}^{+\infty}\left(1+\left|\xi_{n}+i \eta_{n}\right|\right)^{-2} d \xi_{n}\right\}^{1 / 2} \\
\quad \times\left\{\int_{\varepsilon^{-1}<\left|\xi_{n}\right|<2 \varepsilon^{-1}} d \xi_{n}\right\}^{1 / 2} \exp \left\{\left(y_{n}-x_{n}\right) \eta_{n}\right\} \\
\leq C \sqrt{\varepsilon}\left(1+\left|\xi^{\prime}+i \eta^{\prime}\right|\right)^{k} \exp \left\{\left(y_{n}-x_{n}\right) \eta_{n}\right\},
\end{aligned}
$$

where $C$ and $k$ are some positive constants. Since $\psi \in \mathscr{D}\left(\boldsymbol{R}^{n-1}\right)$ we have for any $N$

$$
\left|\widehat{\psi}\left(\xi^{\prime}+i \eta^{\prime}\right)\right| \leq C_{N}\left(1+\left|\xi^{\prime}+i \eta^{\prime}\right|\right)^{-N} \exp \left\{h_{\psi}\left(\eta^{\prime}\right)\right\},
$$




$$
h_{\psi}\left(\eta^{\prime}\right)=\operatorname{Max}_{x^{\prime} \in \operatorname{supp} \psi} x^{\prime} \cdot \eta^{\prime} .
$$

If we take $\rho$ with support in $\left(-\infty, y_{n}\right) \cup\left(y_{n}, \infty\right)$, we may substitute (6.11) in the right-hand side of (6.4). Since we have (6.13), (6.14) and (6.15), letting $\varepsilon$ tend to zero, we get the following expression by Lebesgue's dominated convergence theorem.

$$
\begin{aligned}
& \left\langle G(x-y), \check{\psi}\left(x^{\prime}\right) \check{\rho}\left(x_{n}\right)\right\rangle \\
& =\int_{-\infty}^{+\infty}\left(y_{n}-x_{n}\right)^{-\alpha} \int_{\nabla^{n-1}} \exp \left\{-i y^{\prime}\left(\xi^{\prime}+i \eta^{\prime}\right)\right\} \\
& \quad \times \widehat{\psi}\left(\xi^{\prime}+i \eta^{\prime}\right)\left[\int_{-\infty}^{+\infty} \exp \left\{i\left(x_{n}-y_{n}\right)\left(\xi_{n}+i \eta_{n}\right)\right\}\right. \\
& \left.\quad \times\left(-i \partial / \partial \xi_{n}\right)^{\alpha}(P(\xi+i \eta))^{-1} d \xi_{n}\right] \rho\left(x_{n}\right) d \xi^{\prime} d x_{n} .
\end{aligned}
$$

Therefore we have for any sufficiently large $\alpha$

$$
\begin{aligned}
& \left\langle G(x-y), \breve{\phi}\left(x^{\prime}\right)\right\rangle \\
& =\left(y_{n}-x_{n}\right)^{-\alpha} \int_{z^{n}} \exp \left\{-i y(\xi+i \eta)-i x_{n}\left(\xi_{n}+i \eta_{n}\right)\right\} \\
& \quad \times \widehat{\psi}\left(\xi^{\prime}+i \eta^{\prime}\right)\left(-i \partial / \partial \xi_{n}\right)^{\alpha}(P(\xi+i \eta))^{-1} d \xi
\end{aligned}
$$

when $x_{n} \neq y_{n}$ and we see that $G(x-y) \in C^{\infty}\left(\left(-\infty, y_{n}\right) ; \mathscr{D}^{\prime}\left(\boldsymbol{R}^{n-1}\right)\right)$ $\cap C^{\infty}\left(\left(y_{n},+\infty\right) ; \mathscr{D}^{\prime}\left(\boldsymbol{R}^{n-1}\right)\right)$. This implies that for any integer $l \geq 0$ $\left.D_{n}^{l} G(x-y)\right|_{x_{n}=0}$ makes sence when $y_{n} \neq 0$. Moreover the estimates (6.6) and (6.15) enable us to differentiate the right side of (6.17) with respect to $x_{n}$ under the integral sign and so $\left.D_{n}^{l} G(x-y)\right|_{x_{n}=0}$ is given in the form

$$
\begin{aligned}
\left\langle\left. D_{n}^{l} G(x-y)\right|_{x_{n}=0}, \breve{\psi}\left(x^{\prime}\right)\right\rangle \\
=\sum_{\nu=0}^{l} C_{\alpha l_{\nu}} y_{n}^{-a-\nu} \int_{g_{n}} \exp \{-i y(\xi+i \eta)\} \widehat{\psi}\left(\xi^{\prime}+i \eta^{\prime}\right) \\
\quad \times\left(\xi_{n}+i \eta_{n}\right)^{l-\nu}\left(-i \partial / \partial \xi_{n}\right)^{\alpha}(P(\xi+i \eta))^{-1} d \xi, \quad y_{n} \neq 0,
\end{aligned}
$$

where $\alpha$ is chosen sufficiently large and $C_{\alpha l_{\nu}}=\alpha(\alpha+1) \cdots(\alpha+\nu-1)\left(\begin{array}{l}l \\ \nu\end{array}\right)$. Writing

$$
B_{j}(D)=\sum_{l=0}^{r_{j}} b_{j l}\left(D^{\prime}\right) D_{n}^{l}
$$

we also find 
(6. 19)

$$
\begin{aligned}
& \left\langle\left. B_{j}(D) G(x-y)\right|_{x_{n}=0}, \check{\psi}\left(x^{\prime}\right)\right\rangle \\
& =\sum_{l=0}^{r_{j}}\left\langle\left. D_{n}^{l} G(x-y)\right|_{x_{n}=0}, b_{j l}\left(-D^{\prime}\right) \check{\psi}\left(x^{\prime}\right)\right\rangle \\
& =\sum_{l=0}^{r_{j}} \sum_{\nu=0}^{l} C_{\alpha l \nu} y_{n}^{-\alpha-\nu} \int_{g_{n}} \exp \{-i y(\xi+i \eta)\} b_{j l}\left(\xi^{\prime}+i \eta^{\prime}\right) \\
& \quad \times \widehat{\psi}\left(\xi^{\prime}+i \eta^{\prime}\right)\left(\xi_{n}+i \eta_{n}\right)^{l-\nu}\left(-i \partial / \partial \xi_{n}\right)^{\alpha}(P(\xi+i \eta))^{-1} d \xi, \\
& y_{n} \neq 0 .
\end{aligned}
$$

Now we define $F\left(\cdot, x_{n}, y\right) \in C^{\infty}\left([0, \infty) ; \mathscr{D}^{\prime}\left(\boldsymbol{R}^{n-1}\right)\right)$ by

$$
\begin{aligned}
& \left\langle F(x, y), \check{\psi}\left(x^{\prime}\right)\right\rangle=(2 \pi)^{-n}(-i) \sum_{j, k=1}^{p} \sum_{l=0}^{r_{j}} \sum_{\nu=0}^{l} C_{\alpha l \nu} y_{n}^{-\alpha-\nu} \\
& \quad \times \int_{\Sigma^{n}}\left\{\oint \frac{\exp \left\{i x_{n} \lambda\right\} \lambda^{k-1}}{P_{+}\left(\xi^{\prime}+i \eta^{\prime}, \lambda\right)} d \lambda\right\} \exp \{-i y(\xi+i \eta)\} \frac{R_{j k}\left(\xi^{\prime}+i \eta^{\prime}\right)}{R\left(\xi^{\prime}+i \eta^{\prime}\right)} \\
& \quad \times b_{j l}\left(\xi^{\prime}+i \eta^{\prime}\right) \hat{\psi}\left(\xi^{\prime}+i \eta^{\prime}\right)\left(\xi_{n}+i \eta_{n}\right)^{l-\nu}\left(-i \partial / \partial \xi_{n}\right)^{k} \\
& \quad \times(P(\xi+i \eta))^{-1} d \xi
\end{aligned}
$$

where $R_{j k}\left(\xi^{\prime}+i \eta^{\prime}\right)$ is the $(j, k)$ cofactor of the matrix

$$
\begin{gathered}
\left((2 \pi i)^{-1} \oint B_{j}\left(\xi^{\prime}+i \eta^{\prime}, \lambda\right) \lambda^{k-1}\left(P_{+}\left(\xi^{\prime}+i \eta^{\prime}, \lambda\right)\right)^{-1} d \lambda\right)_{j, k=1, \ldots, p} \\
\xi^{\prime}+i \eta^{\prime} \in \boldsymbol{\Xi}^{n-1}-i \gamma_{1} \vartheta^{\prime}-i \dot{\Sigma} .
\end{gathered}
$$

If we put

$$
E(x, y)=G(x-y)-F(x, y),
$$

then $E(x, y)$ is the required fundamental solution, that is, $E(x, y)$ belongs to the space $C^{\infty}\left(\left[0, y_{n}\right) ; \mathscr{D}^{\prime}\left(\boldsymbol{R}^{n-1}\right)\right) \cap C^{\infty}\left(\left(y_{n}, \infty\right) ; \mathscr{D}^{\prime}\left(\boldsymbol{R}^{n-1}\right)\right)$ and satisfies the equations

$P\left(D_{x}\right) E(x, y)=\delta(x-y), x_{n}>0, y_{n}>0,\left.B_{j}\left(D_{x}\right) E(x, y)\right|_{x_{n}=0}=0, j=1, \cdots, p$.

From Lemma 4. 4 and the Paley-Wiener-Schwartz theorem, it follows that there exists a closed proper cone $K$ with its vertex at the origin such that $x \cdot \vartheta>0$ on $K-\{0\}$ and $\operatorname{supp}_{x} E(x, y) \subset(K+y) \cap \overline{\boldsymbol{R}_{+}^{n}}$.

On the other hand, the fundamental solutions $F_{j}(x)$ are defined by (6. 21) $\left\langle F_{j}(x), \check{\psi}\left(x^{\prime}\right)\right\rangle$

$$
=(2 \pi)^{-n}(-i) \sum_{k=1}^{p} \int_{g_{n-1}}\left\{\oint \exp \left\{i x_{n} \lambda\right\} \lambda^{k-1}\left(P_{+}\left(\xi^{\prime}+i \eta^{\prime}, \lambda\right)\right)^{-1} d \lambda\right\}
$$




$$
\times R_{j k}\left(\xi^{\prime}+i \eta^{\prime}\right)\left(R\left(\xi^{\prime}+i \eta^{\prime}\right)\right)^{-1} \widehat{\psi}\left(\xi^{\prime}+i \eta^{\prime}\right) d \xi^{\prime}, \quad j=1, \cdots, p .
$$

Obviously the $F_{j}(x), j=1, \cdots, p$ belong the space $C^{\infty}\left([0, \infty), \mathscr{D}^{\prime}\left(\boldsymbol{R}^{n-1}\right)\right)$ and satisfy the equations:

$$
P(D) F_{j}(x)=0, x_{n}>0,\left.B_{j}(D) F_{k}(x)\right|_{x_{n}=0}=\delta_{j k} \delta\left(x^{\prime}\right), \quad j, k=1, \cdots, p .
$$

From Lemma 4.4 and the Paley-Wiener-Schwartz theorem we also see that the support of every $F_{j}(x)$ is contained in some closed proper cone having no points $\neq 0$ in common with the half-space $x \cdot \vartheta>0$. Thus we obtained a system $\left\{E(x, y) ; F_{j}(x), j=1, \cdots, p\right\}$ of fundamental solutions of the mixed problem (1.3)-(1.5) for $\left\{P(D) ; B_{j}(D), j=1, \cdots, q\right\}$ which satisfy the required condition on their supports. It only remains to show the uniqueness for such a system.

Proof of the uniqueness of $\left\{\boldsymbol{E}(\boldsymbol{x}, \boldsymbol{y}) ; \boldsymbol{F}_{\boldsymbol{j}}(\boldsymbol{x}), \boldsymbol{j}=\mathbb{1}, \cdots, \boldsymbol{p}\right\}$. The uniqueness of the system $\left\{F_{j}(x), j=1, \cdots, p\right\}$ follows immediately from Proposition 5.1. In fact, suppose that there exists an another system $\left\{F_{j}^{\prime}(x) \in C^{\infty}\left([0, \infty) ; \mathscr{D}^{\prime}\left(\boldsymbol{R}_{x^{\prime}}^{n-1}\right)\right), j=1, \cdots, p\right\}$ satisfying (1.10), (1.11) and the required condition on support. We may assume that supp $F_{j}^{\prime} \subset K$ replacing $K$ by a larger one if necessary. Then $W(x)=F_{j}(x)-F_{j}^{\prime}(x)$ satisfies (5.1), (5.2) and supp $W \subset K$. Therefore we have $F_{j}(x) \equiv F_{j}^{\prime}(x)$ by Proposition 5.1 .

If the hyperplane $x_{n}=0$ is non-characteristic for $P(D)$, the uniqueness of $E(x, y)$ also follows immediately from Proposition 3.1 and Proposition 5.1. First we note that if $u \in \mathscr{D}^{\prime}\left(\boldsymbol{R}_{+}^{n}\right)$ belongs to the space $C^{\infty}\left([0, \delta), \mathscr{D}^{\prime}\left(\boldsymbol{R}_{x^{\prime}}^{n-1}\right)\right)$ for some $\delta>0$, then $u \in \mathscr{D}^{\prime}\left(\overline{\boldsymbol{R}_{+}^{n}}\right)$. In fact, take $\chi\left(x_{n}\right) \in C_{0}^{\infty}((-\delta / 2, \delta / 2))$ with $\chi\left(x_{n}\right)=1$ on $[-\delta / 4, \delta / 4]$ and decompose $u=u+(1-\chi) u \equiv u_{1}+u_{2}$. Then $u_{1} \in C^{\infty}\left([0, \delta) ; \mathscr{D}^{\prime}\left(\boldsymbol{R}_{x^{\prime}}^{n-1}\right)\right)$ and supp $u_{1}$ $\subset \boldsymbol{R}_{x^{\prime}}^{n-1} \times[0, \delta / 2)$ so $u_{1} \in C^{\infty}\left([0, \infty) ; \mathscr{D}^{\prime}\left(\boldsymbol{R}_{x^{\prime}}^{n-1}\right)\right) \subset \mathscr{D}^{\prime}\left(\overline{\boldsymbol{R}_{+}^{n}}\right)$. Since supp $u_{2}$ $\subset \boldsymbol{R}_{x^{\prime}}^{n-1} \times(\delta / 4, \infty)$, we have $u_{2} \in \mathscr{D}^{\prime}\left(\overline{\boldsymbol{R}_{+}^{n}}\right)$ and so $u \in \mathscr{D}^{\prime}\left(\overline{\boldsymbol{R}_{+}^{n}}\right)$. Now suppose that there exists a $E^{\prime}(x, y) \in \mathscr{D}^{\prime}\left(\boldsymbol{R}_{+}^{n}\right) \cap C^{\infty}\left(\left[0, y_{n}\right) ; \mathscr{D}^{\prime}\left(\boldsymbol{R}_{x^{\prime}}^{n-1}\right)\right)$ $\cap C^{\infty}\left(\left(y_{n}, \infty\right) ; \mathscr{D}^{\prime}\left(\boldsymbol{R}_{x^{\prime}}^{n-1}\right)\right)$ satisfying (1.8), (1.9) and $\operatorname{supp}_{x} E^{\prime}(x, y)$ $\subset K+y$. Then $W(x)=E(x, y)-E^{\prime}(x, y) \in \mathscr{D}^{\prime}\left(\overline{\mathbb{R}_{+}^{n}}\right)$ and satisfies (5.1), (5. 2). Hence $W \in C^{\infty}\left([0, \infty) ; \mathscr{D}^{\prime}\left(\boldsymbol{R}_{x^{\prime}}^{n-1}\right)\right)$ by Proposition 3.1. Since supp $W \subset K+y$, it follows from Proposition 5.1 that $W(x) \equiv 0$, that is, $E(x) \equiv E^{\prime}(x, y)$. 
When the hyperplane $x_{n}=0$ is characteristic for $P(D)$, our argument above does not go through. So we shall prove the uniqueness of $E(x, y)$ by a standard method employing the adjoint system of $\{P(D)$; $\left.B_{j}(D), j=1, \cdots, p\right\}$. The following lemma is a modification of Green's formula given in Sakamoto [9].

Lemma 6. 1. Suppose that the assumptions (I), (II) and (III) stated at the beginning of this section hold, and that $u(x)$ is an $\mathcal{E}^{\prime}\left(\boldsymbol{R}_{x^{\prime}}^{n-1}\right)$-valued $C^{\infty}$ function of $x_{n}$ in $[0, \infty)$ with compact support. Then for any $\phi \in C_{0}^{\infty}\left(\boldsymbol{R}_{+}^{n}\right)$ there exist suitable functions $\phi_{j} \in C^{\infty}\left(\boldsymbol{R}_{x^{\prime}}^{n-1}\right) \quad(j=1, \cdots, p), \rho_{j} \in C^{\infty}\left(\boldsymbol{R}_{x^{\prime}}^{n-1}\right) \quad\left(j=1, \cdots, r-m^{\prime}\right)$ and $\phi \in$ $C^{\infty}\left(\overline{\boldsymbol{R}_{+}^{n}}\right)$ such that $\operatorname{supp} \psi \subset \operatorname{supp} \phi+\Lambda, \operatorname{supp} \psi_{j}$, supp $\rho_{j} \subset\left\{x^{\prime} ;\left(x^{\prime}, 0\right) \in \operatorname{supp} \psi\right\}$ and that

$$
\begin{aligned}
& \int_{0}^{\infty}\left\langle P(D) u\left(x^{\prime}, x_{n}\right), \overline{\phi\left(x^{\prime}, x_{n}\right)}\right\rangle d x_{n} \\
& \quad-\int_{0}^{\infty}\left\langle u\left(x^{\prime}, x_{n}\right), \overline{\phi\left(x^{\prime}, x_{n}\right)}\right\rangle d x_{n} \\
& =\sum_{j=1}^{p}\left\langle\left. B_{j}(D) u\right|_{x_{n}=0}, \overline{\left.\psi_{j}\left(x^{\prime}\right)\right\rangle}+\sum_{k=1}^{r-m^{\prime}}\left\langle\left. D_{n}^{k} P(D) u\right|_{x_{n}=0}, \overline{\left.\rho_{k}\left(x^{\prime}\right)\right\rangle},\right.\right.
\end{aligned}
$$

where $\Lambda$ is a closed proper cone such that $x \cdot(-\vartheta)>0$ on $\Lambda-\{0\}, m^{\prime}$ is the order of $P(D)$ in $D_{n}, r$ is the maximum of the orders $r_{j}$ of $B_{j}(D)$ and $\langle$,$\rangle denotes the duality between \mathcal{E}^{\prime}\left(\boldsymbol{R}_{x^{\prime}}^{n-1}\right)$ and $\mathcal{E}\left(\boldsymbol{R}_{x^{\prime}}^{n-1}\right)$.

Proof. Let $\lambda_{j}^{+}\left(\zeta^{\prime}\right), j=1, \cdots, p \quad\left(\operatorname{resp} . \lambda_{j}^{-}\left(\zeta^{\prime}\right), j=1, \cdots, m^{\prime}-p\right)$ denote the roots with positive (resp. negative) imaginary part of the equation $P\left(\zeta^{\prime}, \lambda\right)=0$ in $\lambda$ when $\zeta^{\prime} \in \Xi^{n-1}-i \gamma_{0} \vartheta^{\prime}-i \dot{\Gamma}$, where $m^{\prime}$ is the order of $P\left(\xi^{\prime}, \xi_{n}\right)$ in $\xi_{n}$ (see Section 4$)$. Put

$$
P_{+}\left(\zeta^{\prime}, \lambda\right)=\Pi_{j=1}^{p}\left(\lambda-\lambda_{j}^{+}\left(\zeta^{\prime}\right)\right), P_{-}\left(\zeta^{\prime}, \lambda\right)=\Pi_{j=1}^{m^{\prime}-p}\left(\lambda-\lambda_{j}^{-}\left(\zeta^{\prime}\right)\right) .^{9)}
$$

Then

$$
P\left(\zeta^{\prime}, \lambda\right)=\sum_{j=0}^{m^{\prime}} q_{j}\left(\zeta^{\prime}\right) \lambda^{m^{\prime}-j}=q_{0}\left(\zeta^{\prime}\right) P_{+}\left(\zeta^{\prime}, \lambda\right) P_{-}\left(\zeta^{\prime}, \lambda\right) .
$$

As stated in Section 4, we have

$$
q_{0}\left(\zeta^{\prime}\right) \neq 0 \quad \text { in } \quad \boldsymbol{\Xi}^{n-1}-i \gamma_{0} \vartheta^{\prime}-i \dot{\Gamma} .
$$

9) This notation is different from one in Section 4. 
We write

$$
\begin{aligned}
& P_{+}\left(\zeta^{\prime}, \lambda\right)=\lambda^{p}+a_{1}^{+}\left(\zeta^{\prime}\right) \lambda^{p-1}+\cdots+a_{p}^{+}\left(\zeta^{\prime}\right), \\
& P_{-}\left(\zeta^{\prime}, \lambda\right)=\lambda^{m^{\prime}-p}+a_{1}^{-}\left(\zeta^{\prime}\right) \lambda^{m^{\prime}-p-1}+\cdots+a_{m^{\prime}-p}^{-}\left(\zeta^{\prime}\right) .
\end{aligned}
$$

When $\zeta^{\prime}$ is fixed, we also put for simplicity

$$
\begin{aligned}
& P(\lambda)=P\left(\zeta^{\prime}, \lambda\right), P_{+}(\lambda)=P_{+}\left(\zeta^{\prime}, \lambda\right), P_{-}(\lambda)=P_{-}\left(\zeta^{\prime}, \lambda\right), \\
& \bar{P}(\lambda)=\overline{q_{0}\left(\zeta^{\prime}\right)} \lambda^{m^{\prime}}+\overline{q_{1}\left(\zeta^{\prime}\right)} \lambda^{m^{\prime}-1}+\cdots+\overline{q_{m^{\prime}}\left(\zeta^{\prime}\right)}, \\
& \bar{P}_{+}(\lambda)=\lambda^{p}+\overline{a_{1}^{+}\left(\zeta^{\prime}\right)} \lambda^{p-1}+\cdots+\overline{a_{p}^{+}\left(\zeta^{\prime}\right)}, \\
& \bar{P}_{-}(\lambda)=\lambda^{m^{\prime}-p}+\overline{a_{1}^{-}\left(\zeta^{\prime}\right)} \lambda^{m^{\prime}-p-1}+\cdots+\overline{a_{m^{\prime}-p}^{-}\left(\zeta^{\prime}\right)}, \\
& (u, v)=\int_{0}^{\infty} u\left(x_{n}\right) \overline{v\left(x_{n}\right)} d x_{n},\langle u, v\rangle=u(0) \overline{v(0)},
\end{aligned}
$$

and

$$
\mathscr{F}_{x^{\prime}}[u]\left(\zeta^{\prime}, x_{n}\right)=\hat{u}\left(\zeta^{\prime}, x_{n}\right)=u\left(x_{n}\right) .
$$

With this notation we have

$$
\begin{aligned}
& \left(P\left(D_{n}\right) u, v\right)-\left(u, \bar{P}\left(D_{n}\right) v\right) \\
& =i \sum_{k=0}^{m^{\prime}-p-1} q_{0}\left(\zeta^{\prime}\right) a_{k}^{-}\left(\zeta^{\prime}\right)\left\{\left\langle D_{n}^{m^{\prime}-p-k-1} P_{+}\left(D_{n}\right) u, v\right\rangle+\cdots\right. \\
& \left.\quad \cdots+\left\langle P_{+}\left(D_{n}\right) u, D_{n}^{m^{\prime}-p-k-1} v\right\rangle\right\} \\
& \quad+i \sum_{k=0}^{p-1} q_{0}\left(\zeta^{\prime}\right) a_{k}^{+}\left(\zeta^{\prime}\right)\left\{\left\langle D_{n}^{p-k-1} u, \bar{P}_{-}\left(D_{n}\right) v\right\rangle+\cdots\right. \\
& \left.\quad \cdots+\left\langle u, D_{n}^{p-k-1} \bar{P}_{-}\left(D_{n}\right) v\right\rangle\right\}
\end{aligned}
$$

Now we set

$$
\begin{aligned}
& { }^{t} U=\left(u(0), D_{n} v(0), \cdots, D_{n}^{p-1} u(0),\right. \\
& \left.P_{+}\left(D_{n}\right) u(0), \cdots, D_{n}^{m^{\prime}-p-1} P_{+}\left(D_{n}\right) u(0)\right), \\
& { }^{t} V=\left(D_{n}^{p-1} \bar{P}_{-}\left(D_{n}\right) v(0), \cdots, \bar{P}_{-}\left(D_{n}\right) v(0), D_{n}^{m^{\prime}-p-1} v(0), \cdots, v(0)\right) \text {, }
\end{aligned}
$$

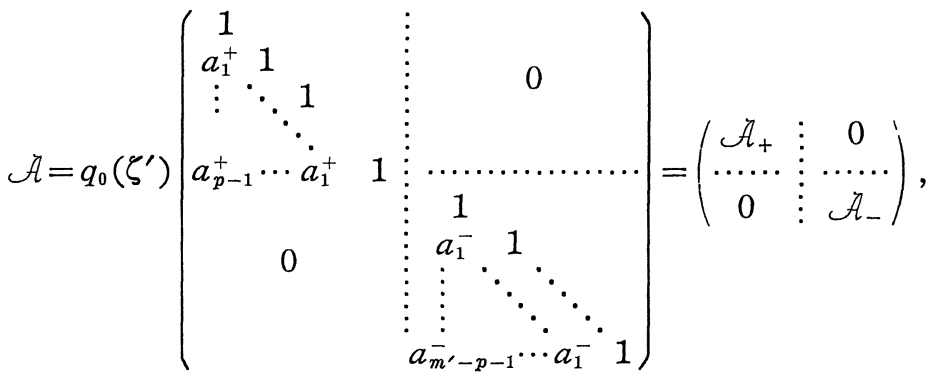


where ${ }^{t} U$ denotes the transpose of $U$, then we have

$$
\left(P\left(D_{n}\right) u, v\right)-\left(u, \bar{P}\left(D_{n}\right) v\right)=i V^{*} \not{A} U,
$$

where $V^{*}$ denotes the adjoint of $V$. Put

$$
B_{j}\left(\zeta^{\prime}, \lambda\right)=Q_{j}\left(\zeta^{\prime}, \lambda\right) P\left(\zeta^{\prime}, \lambda\right)+B_{j}^{\prime}\left(\zeta^{\prime}, \lambda\right), j=1, \cdots, m^{\prime}-p,
$$

and

$$
\begin{gathered}
B_{j+p}^{\prime}\left(\zeta^{\prime}, D_{n}\right)=D_{n}^{j-1} P_{+}\left(\zeta^{\prime}, D_{n}\right), j=1, \cdots, m^{\prime}-p, \\
\zeta^{\prime} \in \mathbb{\Xi}^{n-1}-i \gamma_{1} \vartheta^{\prime}-i \dot{\Sigma},
\end{gathered}
$$

where $\operatorname{deg} Q_{j}\left(\zeta^{\prime}, \lambda\right) \leq r-m^{\prime}$ (if $\left.r-m^{\prime}<0, Q_{j}\left(\zeta^{\prime}, \lambda\right)=0\right)$. When $\zeta^{\prime}$ is fixed in $\boldsymbol{\Xi}^{n-1}-i \gamma_{1} \vartheta^{\prime}-i \dot{\Sigma}$ we also put

$$
B_{j}^{\prime}(\lambda)=B_{j}^{\prime}\left(\zeta^{\prime}, \lambda\right) .
$$

Let us denote

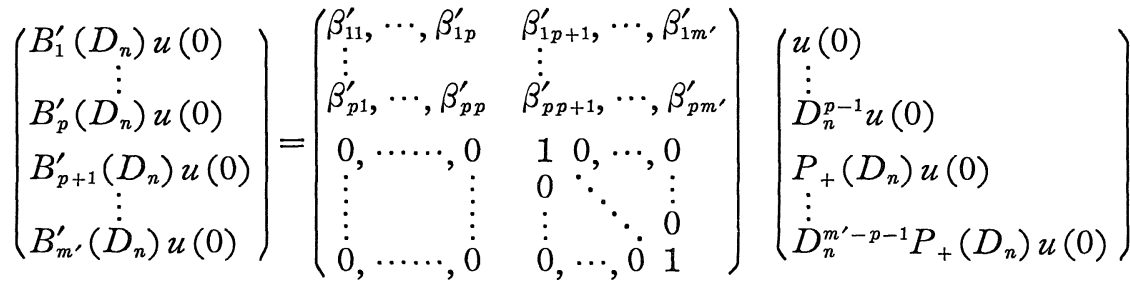

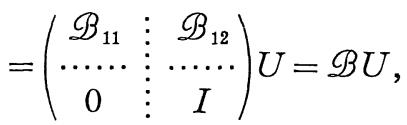

then we have $\operatorname{det} \mathscr{B}=\operatorname{det} \mathscr{B}_{11}=R$, where $R$ is the Lopatinski determinant of the system $\left\{P(D), B_{j}(D), j=1, \cdots, p\right\}$. Since $R=R\left(\zeta^{\prime}\right) \neq 0$ by Lemma 4.4 for $\zeta^{\prime} \in \Xi^{n-1}-i \gamma_{1} \vartheta^{\prime}-i \dot{\Sigma}$, we can put

$$
\mathscr{B}^{\prime \prime}=\left(\mathcal{A} \mathscr{B}^{-1}\right)^{*} \text {, }
$$

and then we have

$$
\left(P\left(D_{n}\right) u, v\right)-\left(u, \bar{P}\left(D_{n}\right) v\right)=i\left(\mathscr{B}^{\prime \prime} V\right)^{*}(\mathscr{B} U) .
$$

Put

(6. 26) $\quad \mathscr{B}^{\prime \prime} V=\left(\begin{array}{l}B_{1}^{\prime \prime}\left(\overline{\zeta^{\prime}}, D_{n}\right) v(0) \\ B_{p}^{\prime \prime}\left(\overline{\zeta^{\prime}}, D_{n}\right) v(0) \\ B_{p+1}^{\prime \prime}\left(\overline{\zeta^{\prime}}, D_{n}\right) v(0) \\ \left.B_{m}^{\prime \prime}, \overline{\zeta^{\prime}}, D_{n}\right) v(0)\end{array}\right)$ 


$$
\begin{aligned}
& \left(\begin{array}{cc}
b_{11}^{\prime \prime}\left(\overline{\zeta^{\prime}}\right), \cdots, b_{1 p}^{\prime \prime}\left(\overline{\zeta^{\prime}}\right) & b_{1 p+1}^{\prime \prime}\left(\overline{\zeta^{\prime}}\right), \cdots, b_{1 m^{\prime}}^{\prime \prime}\left(\overline{\zeta^{\prime}}\right) \\
\vdots \bar{\vdots} & \vdots \\
\left.b_{p 1}^{\prime \prime} \overline{\left(\zeta^{\prime}\right.}\right), \cdots, b_{p p}^{\prime \prime}\left(\overline{\zeta^{\prime}}\right) & b_{p p+1}^{\prime \prime}\left(\overline{\zeta^{\prime}}\right), \cdots, b_{p m^{\prime}}^{\prime \prime}\left(\overline{\zeta^{\prime}}\right) \\
b_{p+11}^{\prime \prime}\left(\overline{\zeta^{\prime}}\right), \cdots, b_{p+1 p}^{\prime \prime}\left(\overline{\zeta^{\prime}}\right) & b_{p+1 p+1}^{\prime \prime}\left(\overline{\zeta^{\prime}}\right), \cdots, b_{p+1 m^{\prime}}^{\prime \prime}\left(\overline{\zeta^{\prime}}\right) \\
\vdots & \vdots \\
b_{m}^{\prime \prime}\left(\overline{\zeta^{\prime}}\right), \cdots, b_{m p}^{\prime \prime}\left(\overline{\zeta^{\prime}}\right) & b_{m^{\prime} p+1}^{\prime \prime}\left(\overline{\zeta^{\prime}}\right), \cdots, b_{m^{\prime} m^{\prime}}^{\prime \prime}\left(\overline{\zeta^{\prime}}\right)
\end{array}\right) V \\
& =\left(\begin{array}{cc}
\mathcal{B}_{11}^{\prime \prime} & \mathcal{B}_{12}^{\prime \prime} \\
\mathcal{B}_{21}^{\prime \prime} & \mathcal{B}_{22}^{\prime \prime}
\end{array}\right) V .
\end{aligned}
$$

Note that if $f\left(\zeta^{\prime}\right)$ is holomorphic in $\sigma^{n-1}-i \gamma_{1} \vartheta^{\prime}-i \dot{\Sigma}$, then $\overline{f\left(\zeta^{\prime}\right)}$ is holomorphic in $\boldsymbol{E}^{n-1}+i \gamma_{1} \vartheta^{\prime}+i \dot{\Sigma}$. The coefficients of $B_{j}^{\prime \prime}\left(\overline{\zeta^{\prime}}, \lambda\right)$ are all holomorphic in $\boldsymbol{\Xi}^{n-1}+i \gamma_{1} \vartheta^{\prime}+i \dot{\Sigma}$. Hence, it follows from (6.23), (6.24) and (6.26) that

$$
\begin{aligned}
\int_{0}^{\infty} P & \left(\zeta^{\prime}, D_{n}\right) \hat{u}\left(\zeta^{\prime}, x_{n}\right) \overline{v\left(x_{n}\right)} d x_{n} \\
& \quad-\int_{0}^{\infty} \hat{u}\left(\zeta^{\prime}, x_{n}\right) \overline{\bar{P}\left(\overline{\zeta^{\prime}}, D_{n}\right) v\left(x_{n}\right)} d x_{n} \\
= & i \sum_{j=1}^{p} B_{j}\left(\zeta^{\prime}, D_{n}\right) \hat{u}\left(\zeta^{\prime}, 0\right) \cdot \overline{B_{j}^{\prime \prime}\left(\overline{\zeta^{\prime}}, D_{n}\right) v(0)} \\
& -i \sum_{j=1}^{p} \sum_{k=0}^{r-m^{\prime}} D_{n}^{k} P\left(\zeta^{\prime}, D_{n}\right) \hat{u}\left(\zeta^{\prime}, 0\right) \cdot \overline{\overline{q_{j}^{k}\left(\zeta^{\prime}\right)} B_{j}^{\prime \prime}\left(\overline{\zeta^{\prime}}, D_{n}\right) v(0)} \\
& +i \sum_{j=p+1}^{m^{\prime}} B_{j}^{\prime}\left(\zeta^{\prime}, D_{n}\right) \hat{u}\left(\zeta^{\prime}, 0\right) \cdot \overline{B_{j}^{\prime \prime}\left(\overline{\zeta^{\prime}}, D_{n}\right) v(0)}
\end{aligned}
$$

where $Q_{j}\left(\zeta^{\prime}, \lambda\right)=\sum_{k=0}^{r-m^{\prime}} q_{j}^{k}\left(\zeta^{\prime}\right) \lambda^{k}$ (if $r-m^{\prime}<0$ for some $j, q_{j}^{k}\left(\zeta^{\prime}\right)=0$ ) and the coefficients of $B_{j}^{\prime \prime}\left(\overline{\zeta^{\prime}}, D_{n}\right)$ and $\overline{q_{j}^{k}\left(\zeta^{\prime}\right)}$ are all holomorphic in $\overline{\zeta^{\prime}} \in \mathbb{E}^{n-1}+i \gamma_{1} \vartheta^{\prime}+i \dot{\Sigma}$. Since $\bar{P}\left(\overline{\zeta^{\prime}}, \lambda\right)=\overline{q_{0}\left(\zeta^{\prime}\right)} \cdot \bar{P}_{+}\left(\zeta^{\prime}, \lambda\right) \bar{P}_{-}\left(\zeta^{\prime}, \lambda\right)$ when $\overline{\zeta^{\prime}} \in \mathbb{G}^{n-1}+i \gamma_{1} \vartheta^{\prime}+i \dot{\Sigma}$, we denote the roots of the equation $\bar{P}\left(\overline{\zeta^{\prime}}, \lambda\right)=0$ in $\lambda$ by $\tilde{\lambda}_{1}^{+}\left(\overline{\zeta^{\prime}}\right), \cdots, \tilde{\lambda}_{m^{\prime}-p}^{+}\left(\overline{\zeta^{\prime}}\right), \tilde{\lambda}_{1}^{-}\left(\overline{\zeta^{\prime}}\right), \cdots, \tilde{\lambda}_{p}^{-}\left(\overline{\zeta^{\prime}}\right)$, which are enumerated so that $\operatorname{Im} \tilde{\lambda}_{k}^{+}\left(\overline{\zeta^{\prime}}\right)>0, k=1, \cdots, m^{\prime}-p, \operatorname{Im} \tilde{\lambda}_{k}^{-}\left(\overline{\zeta^{\prime}}\right)<0, k=1, \cdots, p$, $\overline{\zeta^{\prime}} \in \boldsymbol{G}^{n-1}+i \gamma_{1} \vartheta^{\prime}+i \dot{\Sigma}$, and then we have

$$
\begin{aligned}
& \bar{P}_{+}\left(\zeta^{\prime}, \lambda\right)=\lambda^{p}+\sum_{j=1}^{p} \overline{a_{j}^{+}\left(\zeta^{\prime}\right)} \lambda^{p-j}=\prod_{j=1}^{p}\left(\lambda-\tilde{\lambda}_{j}^{-}\left(\overline{\zeta^{\prime}}\right)\right) \\
& \bar{P}_{-}\left(\zeta^{\prime}, \lambda\right)=\lambda^{m^{\prime}-p}+\sum_{j=1}^{m^{\prime}-p} \overline{a_{j}^{-}\left(\zeta^{\prime}\right)} \lambda^{m^{\prime}-p-j}=\Pi_{j=1}^{m^{\prime}-p}\left(\lambda-\tilde{\lambda}_{j}^{+}\left(\overline{\zeta^{\prime}}\right)\right), \\
& \overline{\zeta^{\prime}} \in \bar{G}^{n-1}+i \gamma_{1} \vartheta^{\prime}+i \dot{\Sigma} .
\end{aligned}
$$

Put

$$
R^{*}\left(\overline{\zeta^{\prime}}\right)=\operatorname{det}\left((2 \pi i)^{-1} \oint B_{j+p}^{\prime \prime}\left(\overline{\zeta^{\prime}}, \lambda\right) \lambda^{k-1}\left(\bar{P}_{-}\left(\zeta^{\prime}, \lambda\right)\right)^{-1} d \lambda\right)_{j, k=1, \ldots, m^{\prime}-p}
$$




$$
\overline{\zeta^{\prime}} \in \mathbb{E}^{n-1}+i \gamma_{1} \vartheta^{\prime}+i \dot{\Sigma} .
$$

Since

$$
\begin{aligned}
B_{p+j}^{\prime \prime}\left(\overline{\zeta^{\prime}}, \lambda\right)= & b_{p+j 1}^{\prime \prime}\left(\overline{\zeta^{\prime}}\right) \lambda^{p-1} \bar{P}_{-}\left(\zeta^{\prime}, \lambda\right)+\cdots+b_{p+j p}^{\prime \prime}\left(\overline{\zeta^{\prime}}\right) \bar{P}_{-}\left(\zeta^{\prime}, \lambda\right) \\
& +b_{p+j p+j}^{\prime \prime}\left(\overline{\zeta^{\prime}}\right) \lambda^{m^{\prime}-p-1}+\cdots+b_{p+j m^{\prime}}^{\prime \prime}\left(\overline{\zeta^{\prime}}\right),
\end{aligned}
$$

we have

$$
R^{*}\left(\overline{\zeta^{\prime}}\right)=\operatorname{det} \mathscr{B}_{22}^{\prime \prime}, \overline{\zeta^{\prime}} \in \mathbb{G}^{n-1}+i \gamma_{1} \vartheta^{\prime}+i \dot{\Sigma} .
$$

On the other hand, since

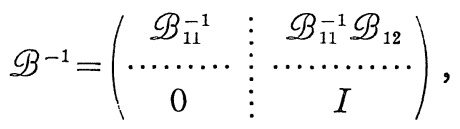

we have $\mathscr{B}_{22}^{\prime \prime}=\left(\mathscr{A}_{-}\right)^{*}$. And then we have $R^{*}\left(\overline{\zeta^{\prime}}\right)=\overline{\left(q_{0}\left(\zeta^{\prime}\right)\right)^{m^{\prime}-p}}$ $\overline{\zeta^{\prime}} \in \mathbb{G}^{n-1}+i \gamma_{1} \vartheta+i \dot{\Sigma}$. Put $R_{j k}^{*}\left(\overline{\zeta^{\prime}}\right)=$ the $(j, k)$ cofactor of

$$
\begin{aligned}
& \left((2 \pi i)^{-1} \oint B_{p+j}^{\prime \prime}\left(\overline{\zeta^{\prime}}, \lambda\right) \lambda^{k-1}\left(\bar{P}_{-}\left(\zeta^{\prime}, \lambda\right)\right)^{-1} d \lambda\right)_{j, k=1, \ldots, m^{\prime}-p}, \\
& \overline{\zeta^{\prime}} \in \boldsymbol{\Xi}^{n-1}+i \gamma_{1} \vartheta^{\prime}+i \dot{\Sigma} .
\end{aligned}
$$

For any $\phi \in C_{0}^{\infty}\left(\boldsymbol{R}_{ \pm}^{n}\right)$ we put

$$
\begin{gathered}
v\left(\xi^{\prime}+i \eta^{\prime}, x_{n}\right)=v_{1}\left(\xi^{\prime}+i \eta^{\prime}, x_{n}\right)-v_{2}\left(\xi^{\prime}+i \eta^{\prime}, x_{n}\right), \\
\xi^{\prime}+i \eta^{\prime} \in \Xi^{n-1}+i \gamma_{1} \vartheta^{\prime}+i \dot{\Sigma}
\end{gathered}
$$

where

$$
\begin{gathered}
v_{1}\left(\xi^{\prime}+i \eta^{\prime}, x_{n}\right)=(2 \pi)^{-1} \int \exp \left\{i x_{n} \xi_{n}\right\} \hat{\phi}\left(\xi^{\prime}+i \eta^{\prime}, \xi_{n}\right) \\
\times\left(\bar{P}\left(\xi^{\prime}+i \eta^{\prime}, \xi_{n}\right)\right)^{-1} d \xi_{n}, \\
v_{2}\left(\xi^{\prime}+i \eta^{\prime}, x_{n}\right)=\sum_{j, k=1}^{m \prime-p}(2 \pi i)^{-1} \oint \exp \left\{i x_{n} \lambda\right\} R_{j k}^{*}\left(\xi^{\prime}+i \eta^{\prime}\right) \lambda^{k-1}\left(R^{*}\left(\xi^{\prime}+i \eta^{\prime}\right)\right)^{-1} \\
\times B_{j+p}^{\prime \prime}\left(\xi^{\prime}+i \eta^{\prime}, D_{n}\right) v_{1}\left(\xi^{\prime}+i \eta^{\prime}, 0\right)\left(\bar{P}_{-}\left(\xi^{\prime}-i \eta^{\prime}, \lambda\right)\right)^{-1} d \lambda, \\
\xi^{\prime}+i \eta^{\prime} \in \Xi^{n-1}+i \gamma_{1} \vartheta^{\prime}+i \dot{\Sigma} .
\end{gathered}
$$

Put

(6. 28) $\quad \psi(x)=(2 \pi)^{-(n-1)} \int_{\eta^{\prime} \in r_{1} \vartheta+\dot{\Sigma}} \exp \left\{i x^{\prime}\left(\xi^{\prime}+i \eta^{\prime}\right)\right\} v\left(\xi^{\prime}+i \eta^{\prime}, x_{n}\right) d \xi^{\prime}$ 
Since $\overline{P(\zeta)} \neq 0$ when $\zeta \in \mathbb{E}^{n}+i \gamma_{0} \vartheta+i \Gamma$ and the coefficients of $\bar{P}_{-}\left(\xi^{\prime}-i \eta^{\prime}\right.$, $\lambda)$ and $B_{p+j}^{\prime \prime}\left(\xi^{\prime}+i \eta^{\prime}, \lambda\right)$ and $R_{j k}^{*}\left(\xi^{\prime}+i \eta^{\prime}\right)$ is holomorphic in $\xi^{\prime}+i \eta^{\prime} \in \boldsymbol{E}^{n-1}$ $+i \gamma_{1} \vartheta^{\prime}+i \dot{\Sigma}$, it follows from Paley-Wiener-Schwartz theorem that $\phi(x)$ belongs to $C^{\infty}\left(\overline{\boldsymbol{R}_{+}^{n}}\right)$, whose support is contained in $\{\operatorname{supp} \phi+\Lambda\} \cap\left\{x_{n} \geq 0\right\}$, where $\Lambda$ is some closed proper cone with its vertex at the origin such that $x \cdot(-\vartheta)>0$ on $\Lambda-\{0\}$. Since $\bar{P}\left(\xi^{\prime}+i \eta^{\prime}, D_{n}\right) v\left(\xi^{\prime}+i \eta^{\prime}, x_{n}\right)$ $=\phi\left(\xi^{\prime}+i \eta^{\prime}, x_{n}\right)$ and $B_{j}^{\prime \prime}\left(\xi^{\prime}+i \eta^{\prime}, D_{n}\right) v\left(\xi^{\prime}+i \eta^{\prime}, 0\right)=0, j=p+1, \cdots, m^{\prime}$, it follows from (6.27) that

$$
\begin{aligned}
& \int_{0}^{\infty} P\left(\xi^{\prime}-i \eta^{\prime}, D_{n}\right) \hat{u}\left(\xi^{\prime}-i \eta^{\prime}, x_{n}\right) \cdot \overline{v\left(\xi^{\prime}+i \eta^{\prime}, x_{n}\right)} d x_{n} \\
& -\int_{0}^{\infty} \hat{u}\left(\xi^{\prime}-i \eta^{\prime}, x_{n}\right) \cdot \overline{\widehat{\phi}\left(\xi^{\prime}+i \eta^{\prime}, x_{n}\right)} d x_{n} \\
& =i \sum_{j=1}^{p} B_{j}\left(\xi^{\prime}-i \eta^{\prime}, D_{n}\right) \hat{u}\left(\xi^{\prime}-i \eta^{\prime}, 0\right) \\
& \cdot \overline{B_{j}^{\prime \prime}\left(\xi^{\prime}+i \eta^{\prime}, D_{n}\right) v\left(\xi^{\prime}+i \eta^{\prime}, 0\right)} \\
& -i \sum_{j=1}^{p} \sum_{k=0}^{r-m^{\prime}} D_{n}^{k} P\left(\xi^{\prime}-i \eta^{\prime}, D_{n}\right) \hat{u}\left(\xi^{\prime}-i \eta^{\prime}, 0\right) \\
& \cdot \overline{q_{j}^{k}\left(\xi^{\prime}-i \eta^{\prime}\right)} B_{j}^{\prime \prime}\left(\xi^{\prime}+i \eta^{\prime}, D_{n}\right) v\left(\xi^{\prime}+i \eta^{\prime}, 0\right) .
\end{aligned}
$$

Put

(6. 30) $\psi_{j}\left(x^{\prime}\right)=(2 \pi)^{-(n-1)} \int_{\eta^{\prime} \in r_{1} \vartheta^{\prime}+\dot{\Sigma}} \exp \left\{i x^{\prime}\left(\xi^{\prime}+i \eta^{\prime}\right)\right\}$

$$
\times B_{j}^{\prime \prime}\left(\xi^{\prime}+i \eta^{\prime}, D_{n}\right) v\left(\xi^{\prime}+i \eta^{\prime}, 0\right) d \xi^{\prime}, \quad j=1, \cdots, p
$$

(6. 31) $\rho_{j}\left(x^{\prime}\right)=(2 \pi)^{-(n-1)} \int_{\eta^{\prime} \in r_{1} v^{\prime}+\dot{\Sigma}} \exp \left\{i x^{\prime}\left(\xi^{\prime}+i \eta^{\prime}\right)\right\}\left\{\sum_{k=1}^{p} \overline{q_{k}^{j}\left(\xi^{\prime}-i \eta^{\prime}\right)}\right.$.

$$
\left.B_{j}^{\prime \prime}\left(\xi^{\prime}+i \eta^{\prime}, D_{n}\right) v\left(\xi^{\prime}+i \eta^{\prime}, 0\right)\right\} d \xi^{\prime}, \quad j=1, \cdots, r-m^{\prime} .
$$

Since the coefficients of $B_{j}^{\prime \prime}\left(\xi^{\prime}+i \eta^{\prime}, D_{n}\right)$ and $\overline{q_{k}^{j}\left(\xi^{\prime}-i \eta^{\prime}\right)}$ are all holomorphic in $\xi^{\prime}+i \eta^{\prime} \in \mathbb{\Xi}^{n-1}+i \gamma_{1} \vartheta^{\prime}+i \dot{\Sigma}$, it follows from the Paley-WienerSchwartz theorem that

$$
\begin{aligned}
& \operatorname{supp} \phi_{j} \subset\left\{x^{\prime} \in \boldsymbol{R}^{n-1} ;\left(x^{\prime}, 0\right) \in \operatorname{supp} \phi\right\} \\
& \operatorname{supp} \rho_{j} \subset\left\{x^{\prime} \in \boldsymbol{R}^{n-1} ;\left(x^{\prime}, 0\right) \in \operatorname{supp} \psi\right\} .
\end{aligned}
$$

We have

$$
\langle u, \bar{v}\rangle=\left\langle u\left(\xi^{\prime}-i \eta^{\prime}, x_{n}\right), \overline{\mathscr{F}}_{x^{\prime}}[\bar{v}]\left(\xi^{\prime}-i \eta^{\prime}, x_{n}\right)\right\rangle,
$$

where 


$$
\begin{aligned}
\overline{\mathscr{F}}_{x^{\prime}}[\bar{v}]\left(\xi^{\prime}-i \eta^{\prime}, x_{n}\right) & =(2 \pi)^{-(n-1)} \int \exp \left\{i x^{\prime}\left(\xi^{\prime}-i \eta^{\prime}\right)\right\} \bar{v}\left(x^{\prime}, x_{n}\right) d x^{\prime} \\
& =(2 \pi)^{-(n-1)} \overline{\int \exp \left\{-i x^{\prime}\left(\xi^{\prime}+i \eta^{\prime}\right)\right\} v\left(x^{\prime}, x_{n}\right) d x^{\prime}} .
\end{aligned}
$$

Hence, it follows from (6.28)-(6.32) that

$$
\begin{aligned}
& \int_{0}^{\infty}\langle P(D) u, \bar{\psi}\rangle d x_{n} \\
& =(2 \pi)^{-(n-1)}\left[\int_{0}^{\infty}\left\langle P\left(\xi^{\prime}-i \eta^{\prime}, D_{n}\right) \hat{u}\left(\xi^{\prime}-i \eta^{\prime}, x_{n}\right), \overline{v\left(\xi^{\prime}+i \eta^{\prime}, x_{n}\right)}\right\rangle d x_{n}\right. \\
& =(2 \pi)^{-2(n-1)} \int_{0}^{\infty}\left\langle P\left(\xi^{\prime}-i \eta^{\prime}, D_{n}\right) \hat{u}\left(\xi^{\prime}-i \eta^{\prime}, x_{n}\right)\right. \text {, } \\
& \int_{\eta^{\prime} \in r_{1} \vartheta^{\prime}+\dot{\Sigma}} \exp \left\{-i x^{\prime}\left(\xi^{\prime}+i \eta^{\prime}\right)\right\} \psi\left(x^{\prime}, x_{n}\right) d x^{\prime}>d x_{n} \\
& =(2 \pi)^{-(n-1)}\left[\int_{0}^{\infty}\left\langle\hat{u}\left(\xi^{\prime}-i \eta^{\prime}, x_{n}\right), \overline{\hat{\phi}\left(\xi^{\prime}+i \eta^{\prime}, x_{n}\right)}\right\rangle d x_{n}\right. \\
& +i \sum_{j=1}^{p}\left\langle B_{j}\left(\xi^{\prime}-i \eta^{\prime}, D_{n}\right) \hat{u}\left(\xi^{\prime}-i \eta^{\prime}, 0\right)\right. \text {, } \\
& \overline{\left.B_{j}^{\prime \prime}\left(\xi^{\prime}+i \eta^{\prime}, D_{n}\right) v\left(\xi^{\prime}+i \eta^{\prime}, 0\right)\right\rangle} \\
& -i \sum_{j=1}^{p} \sum_{k=0}^{r-m^{\prime}}\left\langle D_{n}^{k} P\left(\xi^{\prime}-i \eta^{\prime}, D_{n}\right) \hat{u}\left(\xi^{\prime}-i \eta^{\prime}, 0\right),\right. \\
& \left.\left.\overline{q_{j}^{k}\left(\xi^{\prime}-i \eta^{\prime}\right) \cdot B_{j}^{\prime \prime}\left(\xi^{\prime}+i \eta^{\prime}, D_{n}\right) v\left(\xi^{\prime}+i \eta^{\prime}, 0\right)}\right\rangle\right] \\
& =\int_{0}^{\infty}\langle u(x), \bar{\phi}(x)\rangle d x_{n}+i \sum_{j=1}^{p}\left\langle B_{j}(D) u\left(x^{\prime}, 0\right), \overline{\left.\psi_{j}\left(x^{\prime}\right)\right\rangle}\right. \\
& -i \sum_{j=0}^{r-m^{\prime}}\left\langle D_{n}^{j} P(D) u\left(x^{\prime}, 0\right), \overline{\rho_{j}\left(x^{\prime}\right)}\right\rangle .
\end{aligned}
$$

Q.E.D.

Proposition 6. 2. Under the hypotheses (I), (II) and (III) we have $W(x) \equiv 0$ provided that $W(x)$ is a solution of the homogeneous boundary value problem in $\overline{\boldsymbol{R}_{+}^{n}}$ :

$$
P(D) W(x)=0 \text { in } \overline{\boldsymbol{R}_{+}^{n}},\left.B_{j}(D) W(x)\right|_{x_{n}=0}=0, j=1, \cdots, p,
$$

such that $W(x)$ belongs to $C^{\infty}\left([0, \delta) ; \mathscr{D}^{\prime}\left(R_{x^{\prime}}^{n-1}\right)\right)$ for some $\delta>0$ and that supp $W(x) \subset K \cap \overline{\boldsymbol{R}_{+}^{n}}$.

Proof. It is sufficient to show that 


$$
\langle W, \phi\rangle=0 \quad \text { for any } \phi \in C_{0}^{\infty}\left(\boldsymbol{R}_{+}^{n}\right) .
$$

In fact, if we show (6.33), then we have

$$
\left\langle W\left(x^{\prime}, x_{n}\right), \phi\left(x^{\prime}\right)\right\rangle_{x^{\prime}}=0 \text { for any } \phi \in \mathscr{D}^{\prime}\left(\boldsymbol{R}^{n-1}\right)
$$

when $0<x_{n}<\delta$. $W(x)$ belongs to $C^{\infty}\left([0, \delta) ; \mathscr{D}^{\prime}\left(\boldsymbol{R}_{x^{\prime}}^{n-1}\right)\right)$. Hence

$$
\left\langle W\left(x^{\prime}, 0\right), \phi\left(x^{\prime}\right)\right\rangle_{x^{\prime}}=\lim _{x_{n} \rightarrow+0}\left\langle W\left(x^{\prime}, x_{n}\right), \phi\left(x^{\prime}\right)\right\rangle_{x^{\prime}}=0 .
$$

We shall show (6.33). Let $\psi, \psi_{j}(j=1, \cdots, p)$ and $\rho_{j}\left(j=1, \cdots, r-m^{\prime}\right)$ be functions which are stated in Lemma 6.1 for $\phi \in C_{0}^{\infty}\left(\boldsymbol{R}_{+}^{n}\right)$. Put

$$
\begin{aligned}
& \Omega_{1}=\left\{x^{\prime} \in \boldsymbol{R}^{n-1} ; x_{1} \geq 0,\left(x^{\prime}, x_{n}\right) \in \operatorname{supp} \psi \text { for some } x_{n}\right\} \\
& \Omega_{2}=\left\{x^{\prime} \in \boldsymbol{R}^{n-1} ; \operatorname{dist}\left(x^{\prime}, \Omega_{1}\right) \leq 1\right\}
\end{aligned}
$$

Let $\chi \in C_{0}^{\infty}\left(\Omega_{2} \times(-\delta / 2, \delta / 2)\right)$ be 1 in $\Omega_{1} \times(-\delta / 4, \delta / 4)$. Let $\sigma_{1}\left(x_{n}\right) \in$ $C_{0}^{\infty}((-\delta, \delta))$ be 1 in $(-\delta / 2, \delta / 2)$ and $\sigma_{2}\left(x^{\prime}\right) \in C^{\infty}\left(\boldsymbol{R}^{n-1}\right)$ be 1 in $x_{1} \geq 0$ and 0 in $x_{1} \leq-1 / 2$. Put

$$
\begin{aligned}
\langle\langle P(D) W, \bar{\psi}\rangle= & \int_{0}^{\infty}\left\langle\sigma_{1}\left(x_{n}\right) P(D) W, \sigma_{2}\left(x^{\prime}\right) \bar{\psi}\right\rangle d x_{n} \\
& +\left\langle\left(1-\sigma_{1}\left(x_{n}\right) P(D) W, \sigma_{2}\left(x^{\prime}\right) \bar{\psi}\right\rangle\right.
\end{aligned}
$$

where $\langle$,$\rangle in the first term of the right-hand side denotes the duality$ between $\mathscr{D}^{\prime}\left(\boldsymbol{R}^{n-1}\right)$ and $\mathscr{D}\left(\boldsymbol{R}^{n-1}\right)$ and $\langle$,$\rangle in the second term of the$ right-hand side denotes the duality between $\mathscr{D}^{\prime}\left(\boldsymbol{R}_{+}^{n}\right)$ and $\mathscr{D}\left(\boldsymbol{R}_{+}^{n}\right)$. Since $\chi W$ is a $C_{(o)}^{\infty}([0, \infty))$ function of $x_{n}$ with its value in $\mathcal{E}^{\prime}\left(\boldsymbol{R}_{x^{\prime}}^{n-1}\right)$ and $\sigma_{1}\left(x_{n}\right) \sigma_{2}\left(x^{\prime}\right)=1$ in supp $\chi \cap\left\{x_{1} \geq 0\right\}$ and supp $W \subset\left\{x_{1} \geq 0\right\}$, it follows from Lemma 6.1 that

$$
\begin{aligned}
\int_{0}^{\infty}\left\langle\sigma_{1}\left(x_{n}\right) P(D) \chi W, \sigma_{2}\left(x_{n}\right) \bar{\psi}\right\rangle d x_{n}=\int_{0}^{\infty}\langle P(D) \chi W, \bar{\phi}\rangle d x_{n}=\langle\chi W, \bar{\phi}\rangle \\
\quad+\sum_{j=1}^{p}\left\langle\left. B_{j}(D) \chi W\right|_{x_{n}=0}, \bar{\phi}_{j}\right\rangle+\sum_{k=1}^{r-m}\left\langle\left. D_{n}^{k} P(D) \chi W\right|_{x_{n}=0}, \bar{\rho}_{j}\right\rangle .
\end{aligned}
$$

Since we have

$$
\begin{aligned}
\left.B_{j}(D) \chi W\right|_{x_{n}=0}= & \left.\chi\left(x^{\prime}, 0\right) B_{j}(D) W\right|_{x_{n}=0} \\
& +\left.\sum_{|\alpha| \geq 1}\left(D^{\alpha} \chi / \alpha !\right)\left(x^{\prime}, 0\right) B_{j}^{(\alpha)}(D) W\right|_{x_{n}=0} \\
= & \left.\sum_{|\alpha| \geq 1}\left(D^{\alpha} \chi / \alpha !\right)\left(x^{\prime}, 0\right) B_{j}^{(\alpha)}(D) W\right|_{x_{n}=0},
\end{aligned}
$$


and $\operatorname{supp}\left(D^{\alpha} \chi / \alpha !\right)\left(x^{\prime}, 0\right) \cap \operatorname{supp} \bar{\psi}_{j} \subset\left\{x_{1}<0\right\}$, it follows that

$$
\left\langle\left. B_{j}(D) \chi W\right|_{x_{n}=0}, \bar{\psi}_{j}\right\rangle=0, j=1, \cdots, p .
$$

Since we have

$$
\left.P(D) \chi W\right|_{x_{n}=0}=\left.\sum_{|\alpha| \geq 1}\left(D^{\alpha} \chi / \alpha !\right)\left(x^{\prime}, 0\right) P^{(\alpha)}(D) W\right|_{x_{n}=0}
$$

and $\operatorname{supp}\left(D^{\alpha} \chi / \alpha !\right)\left(x^{\prime}, 0\right) \cap \operatorname{supp} \bar{\rho}_{j} \subset\left\{x_{1}<0\right\}$, it follows that

$$
\left\langle\left. D_{n}^{k} P(D) \chi W\right|_{x_{n}=0}, \bar{\rho}_{j}\right\rangle=0, \quad j=1, \cdots, r-m^{\prime} .
$$

Hence we have

$$
\int_{0}^{\infty}\left\langle\sigma_{1}\left(x_{n}\right) P(D) \chi W, \sigma_{2}\left(x^{\prime}\right) \bar{\psi}\right\rangle d x_{n}=\langle\chi W, \bar{\phi}\rangle .
$$

Since $\operatorname{supp}\left[\sigma_{1}\left(x_{n}\right) P(D)(1-\chi) W\right] \cap \operatorname{supp} \bar{\psi} \subset\left\{x \in \boldsymbol{R}_{+}^{n} ; x_{1} \geq 0, x_{n} \geq \delta / 4\right\}$, it follows that

$$
\begin{aligned}
\int_{0}^{\infty}\left\langle\sigma_{1}\left(x_{n}\right) P(D)(1-\chi) W, \sigma_{2}\left(x^{\prime}\right) \bar{\psi}\right\rangle d x_{n} \\
\quad=\int_{0}^{\infty}\left\langle W, \overline{\left.(1-\chi) \bar{P}(D)\left(\sigma_{1}\left(x_{n}\right) \sigma_{2}\left(x^{\prime}\right) \psi\right)\right\rangle} d x_{n} .\right.
\end{aligned}
$$

Since $\operatorname{supp}\left(1-\sigma_{1}\left(x_{n}\right)\right) \sigma_{2}\left(x^{\prime}\right) \bar{\phi} \subset\left\{x \in \boldsymbol{R}_{+}^{n} ; x_{1}>-1 / 2, x_{n}>\delta / 2\right\}$, it follows that

$$
\begin{aligned}
& \left\langle\left(1-\sigma_{1}\left(x_{n}\right)\right) P(D) W, \sigma_{2}\left(x^{\prime}\right) \bar{\phi}\right\rangle \\
& =\left\langle P(D) W,\left(1-\sigma_{1}\left(x_{n}\right)\right) \sigma_{2}\left(x^{\prime}\right) \bar{\psi}\right\rangle \\
& =\left\langle W, \overline{\bar{P}}(D)\left(1-\sigma_{1}\left(x_{n}\right)\right) \sigma_{2}\left(x^{\prime}\right) \psi\right\rangle=\left\langle W, \overline{\left.\bar{P}(D)\left(1-\sigma_{1}\left(x_{n}\right)\right) \sigma_{2}\left(x^{\prime}\right) \psi\right\rangle}\right. \text {. }
\end{aligned}
$$

Hence we have

$$
\langle P(D) W, \psi\rangle=\langle W, \bar{\phi}\rangle .
$$

On the other hand, since

$$
\begin{aligned}
\int_{0}^{\infty}\left\langle\sigma_{1}\left(x_{n}\right) P(D) W, \sigma_{2}\left(x^{\prime}\right) \bar{\psi}\right\rangle d x_{n} \\
=\int_{0}^{\infty}\left\langle P(D) W, \sigma_{1}\left(x_{n}\right) \sigma_{2}\left(x^{\prime}\right) \bar{\psi}\right\rangle d x_{n}=0,
\end{aligned}
$$

and since

$$
\left\langle\left(1-\sigma_{1}\left(x_{n}\right)\right) P(D) W, \sigma_{2}\left(x^{\prime}\right) \bar{\psi}\right\rangle
$$




$$
=\left\langle P(D) W,\left(1-\sigma_{1}\left(x_{n}\right)\right) \sigma_{2}\left(x^{\prime}\right) \bar{\psi}\right\rangle=0,
$$

(6. 33) follows.

Q.E.D.

Summing up, we have

Theorem 6. 3. Let $P(D)$ and $B_{j}(D), j=1, \cdots, q$ be differential operators of order $m$ and $r_{j}, j=1, \cdots, q$, respectively, with constant coefficients. The following three assertions are equivalent.

(I) There exists a unique system of fundamental solutions $\{E(x, y)$, $\left.F_{j}(x), j=1, \cdots, q\right\}$ satisfying the following equations:

$$
\begin{aligned}
& P(D) E(x, y)=\delta(x-y) \text { in } \boldsymbol{R}_{+}^{n}, y \in \boldsymbol{R}_{+}^{n}, y=\left(0, y_{2} \cdots, y_{n}\right), \\
& \left.B_{j}(D) E(x, y)\right|_{x_{n}=0}=0 \text { in } \boldsymbol{R}^{n-1}, j=1, \cdots, q . \\
& P(D) F_{k}(x)=0 \text { in } \boldsymbol{R}_{+}^{n}, k=1, \cdots, q, \\
& \left.B_{j}(D) F_{k}(x)\right|_{x_{n}=0}=\delta_{j k} \delta\left(x^{\prime}\right) \text { in } \boldsymbol{R}^{n-1}, j, k=1, \cdots, q,
\end{aligned}
$$

such that $E(x, y)$ and $F_{j}(x)$ belong to $C^{\infty}\left([0, \sigma) ; \mathscr{D}^{\prime}\left(\boldsymbol{R}^{n-1}\right)\right) \cap \mathscr{D}^{\prime}\left(\boldsymbol{R}_{+}^{n}\right)$ for some positive number $\sigma$ and there exists some closed proper cone with its vertex at the origin such that $x \cdot \vartheta>0$ on $x \in K-\{0\}$ such that supp $E(x, y)$ contained in $(K+y) \cap \overline{\boldsymbol{R}_{+}^{n}}$ and supp $F_{k}(x)$ is contained in $K \cap \overline{\boldsymbol{R}_{+}^{n}}$.

(II) There exists a unique system of fundamental solutions $\{E(x, y)$, $\left.F_{j}(x), j=1, \cdots, q\right\}$ satisfying the system of equations (6.34)-(6.37) such that $E(x, y)$ belongs to $C^{\infty}\left(\left[0, y_{n}\right) ; \mathscr{D}^{\prime}\left(\boldsymbol{R}^{n-1}\right)\right) \cap C^{\infty}\left(\left(y_{n}, \infty\right)\right.$; $\left.\mathscr{D}^{\prime}\left(\boldsymbol{R}^{n-1}\right)\right) \cap \mathscr{D}^{\prime}\left(\boldsymbol{R}_{+}^{n}\right)$ and $F_{j}(x)$ belong to $C^{\infty}\left([0, \infty) ; \mathscr{D}^{\prime}\left(\boldsymbol{R}^{n-1}\right)\right)$ and the support of $E(x, y)$ is contained in $(K+y) \cap \overline{\boldsymbol{R}_{+}^{n}}$ and the support of $F_{j}(x)$ is contained in $K \cap \overline{\boldsymbol{R}_{+}^{n}}$.

(III) $P(\xi)$ is a hyperbolic polynomial with respect to $\vartheta$ and $q$ equals the number of roots with positive imaginary part of the equation $P\left(\xi^{\prime}-i s \vartheta^{\prime}, \lambda\right)=0$ in $\lambda$ for large enough $s$ and $\xi^{\prime} \in \boldsymbol{\xi}^{n-1}$ and the Lopatinski determinant $R\left(\zeta^{\prime}\right)$ of the system $\left\{P(D), B_{j}(D), j=1, \cdots\right.$, q) is hyperbolic with respect to $\vartheta^{\prime}$. 


\section{References}

[1] Atiyah, M. F., Bott, R., and Gårding, L., Lacunas for hyperbolic differential operators with constant coefficients, Acta Math. 124 (1970), 109-189.

[2] Gårding, L., Hyperbolic partial differential equations with constant coefficients, Acta Math., 85 (1950), 1-62.

[3] - Some trends and problem in linear partial differential equations, Proc. Int. Congr, Math. Edinburgh, (1958), 87-102.

[4] Gunning, R. and Rossi, H., Analytic functions of several complex variables, Prentice Hall, N. J., (1965).

[5] Hersh, R., Boundary conditions for equations of evolution, Arch Rational Mech. Anal., 19 (1964).

[6] - On surface waves with finite and infinite speed of propagation, Arch. Rational Mech. Anal., 19 (1965).

[7] Hörmander, L., Linear partial differential operators, Grundlehrend. Math. Wiss. 116, Springer-Verlag, (1963).

[8] Kasahara, K., On weak well posedness of mixed problems for hyperbolic systems, Publ. RIMS Kyoto Univ., 6 (1970), 503-514.

[9] Sakamoto, R., $\mathcal{E}$-well-posedness for hyperbolic mixed problems with constant coefficients, J. Math. Kyoto Univ., 14 (1974), 93-118.

[10] Seeley, R. T., Extension of $C^{\infty}$ functions defined in a half-space, Proc, Amer. Math. Soc., 15 (1964), 625-626.

[11] Shirota, T., On the propagation speed of hyperbolic mixed boundary conditions, Jour. Fac. Sci. Hokkaido Univ., 22 (1972).

[12] Wakabayashi, S., Analytic wave front sets of the Riemann functions of hyperbolic mixed problems in a quarter-space, Publ. RIMS Kyoto Univ., 11 (1976), 785-807. 
